\title{
Accessing tens-to-hundreds femtoseconds nuclear state lifetimes with low-energy binary heavy-ion reactions
} \author{
C. Fransen ${ }^{4}$, A. Goldkuhle ${ }^{4}$, J. Grebosz ${ }^{1}$, M. N. Harakeh ${ }^{8}$, L. W. Iskra ${ }^{1,3}$, B. Jacquot ${ }^{5}$, A. Karpov ${ }^{9}$, \\ E. Vardaci $^{20}$, B. Wasilewska ${ }^{1}$, O. Wieland ${ }^{3}$, M. Ziebliński ${ }^{1}$, M. Zielińska ${ }^{21}$ \\ ${ }^{1}$ Institute of Nuclear Physics, PAN, 31-342 Kraków, Poland \\ ${ }^{2}$ Dipartimento di Fisica, Università degli Studi di Milano, 20133 Milan, Italy \\ ${ }^{3}$ INFN Sezione di Milano, via Celoria 16, 20133 Milan, Italy \\ ${ }^{4}$ Institut für Kernphysik, Universität zu Köln, 50937 Cologne, Germany \\ 5 GANIL, CEA/DRF-CNRS/IN2P3, Bd. Henri Becquerel, BP 55027, 14076 Caen, France \\ ${ }^{6}$ CNRS/IN2P3, IPHC UMR 7178, 67037 Strasbourg, France \\ ${ }^{7}$ Department of Physics, Science and Art Faculty, Nigde Omer Halisdemir University, Nigde, Turkey \\ ${ }^{8}$ University of Groningen, Groningen, The Netherlands \\ ${ }^{9}$ FLNR, JINR, 141980 Dubna, Russia \\ ${ }^{10}$ Faculty of Physics, University of Warsaw, Warsaw, Poland \\ ${ }^{11}$ Dipartimento di Fisica e Astronomia, Università degli Studi di Padova, 35131 Padua, Italy \\ 12 INFN Sezione di Padova, 35131 Padua, Italy \\ ${ }^{13}$ IPN Orsay Laboratory, Orsay, France \\ ${ }^{14}$ Institut Laue-Langevin (ILL), Grenoble, France \\ ${ }^{15}$ Heavy Ion Laboratory, University of Warsaw, 02-093 Warsaw, Poland \\ 16 Physics Division, Argonne National Laboratory, Argonne, IL 60439, USA \\ 17 Tata Institute of Fundamental Research, Mumbai 400005, India \\ 18 INFN Laboratori Nazionali di Legnaro, 35020 Legnaro, Italy \\ ${ }^{19}$ IFIN-HH, Bucharest, Romania \\ ${ }^{20}$ Università degli Studi di Napoli and INFN sez., Naples, Italy \\ ${ }^{21}$ IRFU/CEA, Université Paris-Saclay, 91191 Gif-sur-Yvette, France
}

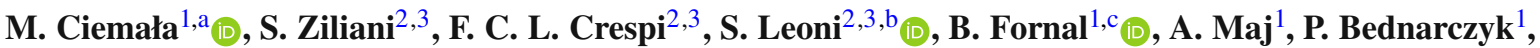

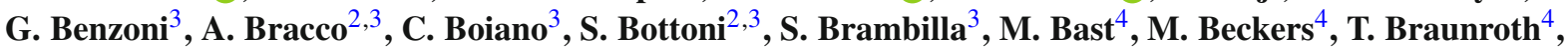

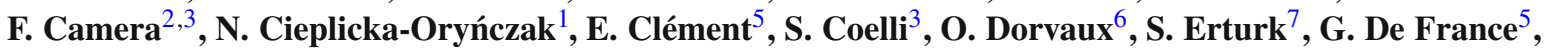
M. Kicińska-Habior ${ }^{10}$, Y.-H. Kim ${ }^{5,14}$, M. Kmiecik ${ }^{1}$, A. Lemasson ${ }^{5}$, S. M. Lenzi ${ }^{11,12}$, M. Lewitowicz ${ }^{5}$ H. Li $^{5}$, I. Matea $^{13}$, K. Mazurek ${ }^{1}$, C. Michelagnoli ${ }^{14}$, M. Matejska-Minda ${ }^{1,15}$, B. Million ${ }^{3}$, C. Müller-Gatermann ${ }^{4,16}$, V. Nanal ${ }^{17}$, P. Napiorkowski ${ }^{15}$, D. R. Napoli ${ }^{18}$, R. Palit ${ }^{17}$, M. Rejmund ${ }^{5}$, Ch. Schmitt ${ }^{6}$, M. Stanoiu ${ }^{19}$, I. Stefan ${ }^{13}$,

Received: 9 December 2020 / Accepted: 29 March 2021 / Published online: 3 May 2021

(C) The Author(s) 2021

Communicated by Wolfram Korten

\begin{abstract}
A novel Monte Carlo technique has been developed to determine lifetimes of excited states in the tensto-hundreds femtoseconds range in products of low-energy heavy-ion binary reactions, with complex velocity distributions. The method is based on a detailed study of Dopplerbroadened $\gamma$-ray lineshapes. Its relevance is demonstrated in connection with the ${ }^{18} \mathrm{O}(7.0 \mathrm{MeV} / \mathrm{u})+{ }^{181} \mathrm{Ta}$ experiment, performed at GANIL with the AGATA+VAMOS+PARIS setup, to study neutron-rich $\mathrm{O}, \mathrm{C}, \mathrm{N}, \ldots$ nuclei. Excited states

\footnotetext{
a e-mail: Michal.Ciemala@ifj.edu.pl (corresponding author)

b e-mail: Silvia.Leoni@mi.infn.it (corresponding author)

${ }^{\mathrm{c}}$ e-mail: Bogdan.Fornal@ifj.edu.pl (corresponding author)
}

in ${ }^{17} \mathrm{O}$ and ${ }^{19} \mathrm{O}$, with known lifetimes, are used to validate the method over the $\sim 20-400$ fs lifetime-sensitivity range. Emphasis is given to the unprecedented position resolution provided by $\gamma$-tracking arrays, which turns out to be essential for reaching the required accuracy in Doppler-shift correction. The technique is anticipated to be an important tool for lifetime investigations in exotic neutron-rich nuclei, produced with intense ISOL-type beams. 


\section{Introduction}

The study of exotic nuclei, i.e., nuclear systems away from the valley of stability, is a central topic in modern nuclear physics. A detailed knowledge of their properties is needed to probe the evolution of the nuclear structure as a function of neutron and proton excess, and to understand the element abundances in the Universe. The heavy-element nucleosynthesis processes in stars [1], as for example the r-process, are in fact to large extent governed by the structural properties of the atomic nuclei involved [2,3]. Detailed experimental investigations are therefore needed in exotic regions of the nuclear chart, which are hard to reach by standard reaction mechanisms. In this context, low-energy binary collisions (which include multi-nucleon transfer and deep-inelastic reactions) [4-9] are considered among the most favorable processes to populate yrast and near-yrast states in nuclei with large neutron excess, when high-intensity radioactive ISOL beams, presently under development, come into operation [10]. With the employment of powerful modern detection systems (for both particles and $\gamma$ rays), high-precision $\gamma$-spectroscopy measurements of very-exotic nuclei will become feasible, yielding nuclear structure information in terms of level energies, spins, parities, state lifetimes, etc.

In this paper, we present a novel approach to access nuclear state lifetimes in the tens-to-hundreds femtoseconds range for products of low-energy binary heavy-ion collisions. In such reactions, the complex structure of the product velocity distribution, caused by large energy dissipation [4-9], does not allow to use standard Doppler-shift attenuation methods employed, for example, in fusion evaporation processes [11]. We note that the short time range of tens-to-hundreds femtoseconds cannot be accessed by relativistic heavy-ion fragmentation, for which typical ranges are few ps to ns $[12,13]$. Therefore, the proposed method offers rather unique opportunities for the investigation of exotic systems with intense stable and radioactive heavy-ion beams.

The technique discussed in this work relies on the highprecision detection capabilities which are now reached with $\gamma$-ray tracking arrays, such as AGATA [14-16] and GRETINA [17,18], coupled to powerful ancillary setups for heavy-ion identification, e.g., the VAMOS++ $[19,20]$, PRISMA [21,22], FMA [23] and S800 [24] spectrometers. The method is based on a recursive procedure which reconstructs the total kinetic energy loss (TKEL) distribution of the reaction, matching the measured velocity distribution after the target. This TKEL distribution is used to simulate the reaction dynamics and the $\gamma$-ray emission. The simulated data are then treated using the same analysis procedure adopted for the $\gamma$-ray tracking and Doppler correction of inbeam data. By varying the energy and the lifetime of the state of interest, the best fit values and their respective uncertainties are found, via a $\chi^{2}$ minimization.
The method has been first applied to extract the lifetime of the second $2^{+}$states in ${ }^{16} \mathrm{C}$ and ${ }^{20} \mathrm{O}$, which have been predicted to be in the hundred-femtoseconds time range and to strongly depend on the three-body term of the nuclear interaction [25]. The experiment was performed at the Grand Accélérateur National d'Ions Lourds (GANIL) in Caen, France, using the AGATA setup coupled to an early implementation of the PARIS scintillator array [26] and to the VAMOS++ heavy-ion spectrometer $[19,20]$. In the measurement, light neutron-rich nuclei of $\mathrm{B}, \mathrm{C}, \mathrm{N}, \mathrm{O}$ and $\mathrm{F}$ were produced in low-energy binary processes induced by an ${ }^{18} \mathrm{O}$ beam on a thick ${ }^{181} \mathrm{Ta}$ target. In the following, the newly developed lifetime analysis will be discussed in detail, in connection with this specific reaction case.

The paper is organized as follows: the experimental setup is presented in Sect. 2, while the analysis of the data is discussed in Sect. 3, focusing on both the heavy-ion identification in the VAMOS++ magnetic spectrometer and the reconstruction of the associated Doppler-shift corrected $\gamma$ ray spectra, measured in the AGATA tracking array. Section 4 describes in details the new lifetime analysis method, based on a Monte Carlo simulation technique. Selected excited states in ${ }^{17} \mathrm{O}$ and ${ }^{19} \mathrm{O}$, with known lifetimes, are considered to validate the technique over the lifetime sensitivity range, i.e., 20-400 fs. The impact of the high precision provided by the AGATA tracking array in identifying the $\gamma$-ray interaction point, which is essential for reaching the required accuracy in Doppler-shift corrections, is also discussed.

\section{Experiment and setup}

In the GANIL experiment, a beam of ${ }^{18} \mathrm{O}$ at $126 \mathrm{MeV}$ (i.e., $7.0 \mathrm{MeV} / \mathrm{u})$ impinging on a ${ }^{181} \mathrm{Ta}$ target $\left(6.64 \mathrm{mg} / \mathrm{cm}^{2}\right.$ thick, tilted at $45^{\circ}$ with respect to the beam direction) was employed to induce direct transfer and deep-inelastic reactions producing a variety of neutron-rich nuclei, from $B(Z=5)$ to $F(Z=9)$,

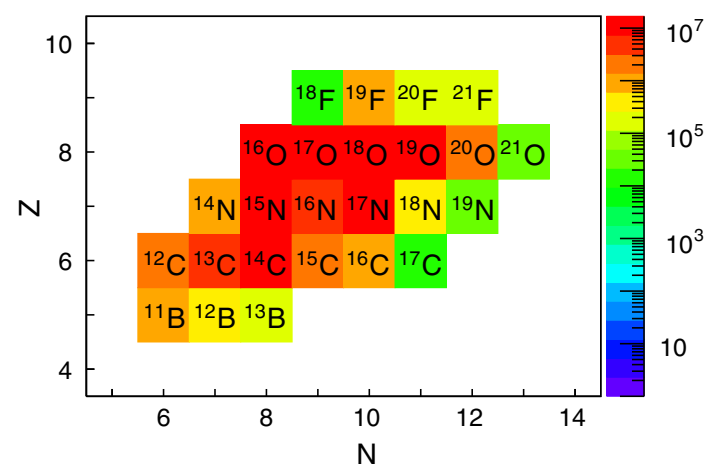

Fig. 1 Population of detected and identified ions in the ${ }^{18} \mathrm{O}(126 \mathrm{MeV})+{ }^{181} \mathrm{Ta}$ collision, investigated at GANIL with the AGATA+PARIS+VAMOS setup [25] 
as shown in Fig. 1 [25]. The beam energy at the center of the target was $\sim 116 \mathrm{MeV}$ (i.e., $\sim 60 \%$ above the Coulomb barrier), and projectile-like products had velocities $v / c \sim 10 \%$, resulting in a target crossing time $T_{\text {cross }} \sim 130 \mathrm{fs}$.

Following the reaction, the $\gamma$ rays emitted by the excited nuclei were detected by the AGATA tracking array [14-16], consisting of 31 segmented High Purity Germanium (HPGe) detectors, coupled to the PARIS scintillators array [26], with two complete clusters of nine phoswich detectors each, plus two large-volume $\left(3.5^{\prime \prime} \times 8^{\prime \prime}\right) \mathrm{LaBr}_{3}$ :Ce scintillators $[29,30]$.

The projectile-like products were detected in the VAMOS++ magnetic spectrometer $[19,20]$, placed at the reaction grazing angle $\theta_{g}=45^{\circ}$, relative to the beam direction, and aligned with the center of AGATA. In this configuration, the VAMOS++ acceptance was $\left(\theta_{a c c}, \phi_{a c c}\right) \sim( \pm 120, \pm 200)$ $\operatorname{mrad}\left(\right.$ i.e.,$\left.\sim\left( \pm 6.9^{\circ}, \pm 11.5^{\circ}\right)\right)$, considering the center of the spectrometer in $(0,0)$. The PARIS array was placed at $90^{\circ}$, with respect to the VAMOS++ axis, while AGATA covered the angular range between $\sim 115^{\circ}$ and $\sim 175^{\circ}$. A schematic drawing and a picture of the experimental setup are shown in Fig. 2.

\section{Data processing}

In the following sections, the processing of the data, collected in the ${ }^{18} \mathrm{O}+{ }^{181} \mathrm{Ta}$ experiment, is discussed in detail. Section 3.1 is devoted to the VAMOS++ magnetic spectrometer, which allows to identify the atomic number $\mathrm{Z}$ and the mass A of the ions, and to precisely reconstruct their trajectories. Fine corrections, which can be applied to the masses and beam-spot reconstruction, taking advantage of recently implemented VAMOS++ entrance detectors and of the fast scintillators of PARIS, are also discussed. Section 3.2 focuses instead on the offline processing of the AGATA data.

\subsection{Ions identification and trajectory reconstruction in VAMOS++}

The focal plane of the VAMOS++ spectrometer consisted of: (i) four drift chambers for the reconstruction of the $\theta, \phi$ scattering angles of the reaction product, (ii) a segmented ionization chamber, divided into six columns and four rows, for measuring the ion energy loss $\Delta \mathrm{E}$, and (iii) one long plastic scintillator at the end of the focal plane, giving the trigger signal, the particle residual energy $\mathrm{E}$ and the time with respect to the cyclotron radiofrequency (RF). Two additional pairs of drift chambers (DC) were also placed at the entrance of VAMOS++, at $20 \mathrm{~cm}$ distance from the target, in order to accurately determine the $\theta$ and $\phi$ angles of emission of the light ions, with good efficiency [27]. They also significantly improved the $\gamma$-ray Doppler-shift correction. Figures $3 \mathrm{a}, \mathrm{b}$ show comparisons of the reconstructed $\theta$ and $\phi$ (a)

VAMOS

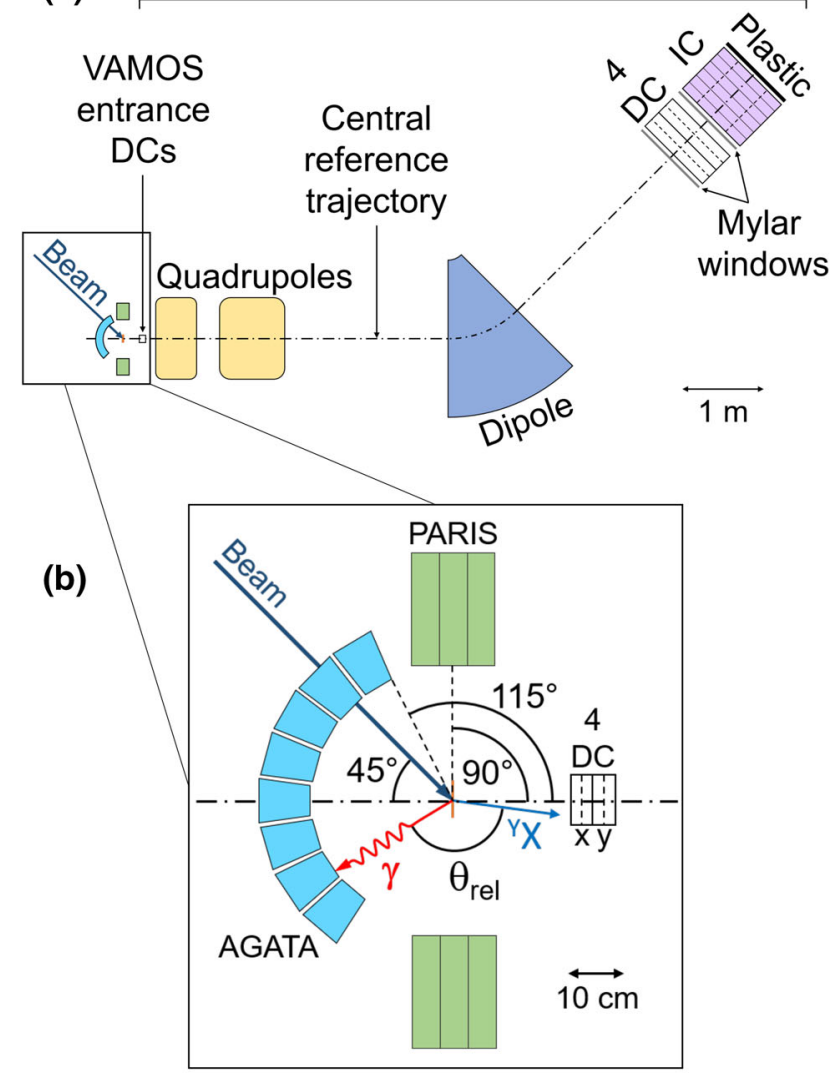

(c)

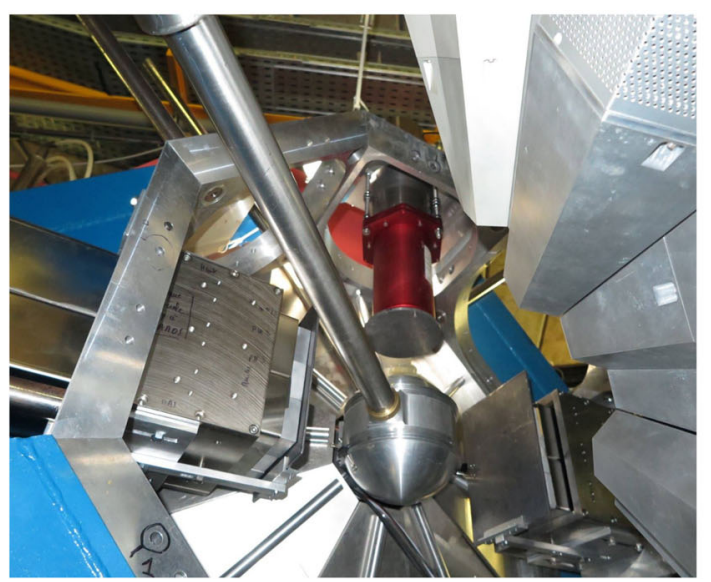

Fig. 2 Panel a: overall view of the experimental setup. The main optical elements of the VAMOS++ spectrometer (i.e., the quadrupole and dipole magnets) are shown, together with the entrance and focal plane detectors. Panel b: zoom of the AGATA+PARIS detectors around the reaction point. The entrance detectors of VAMOS++ (four drift chambers, for $x$ and $y$ position identification) are also shown. Panel c: picture of the AGATA and scintillator arrays. The spherical scattering chamber is in the middle, surrounded by the two PARIS clusters, and the two large-volume $\mathrm{LaBr}_{3}$ :Ce scintillators (one at the top, in red) and the AGATA detectors (on the right) (see text for details)

angles, respectively, using VAMOS++ focal plane only, and the entrance DCs. It is seen that the $\theta$ angle is well determined by VAMOS++ (a), while this is not the case for the $\phi$ angle 

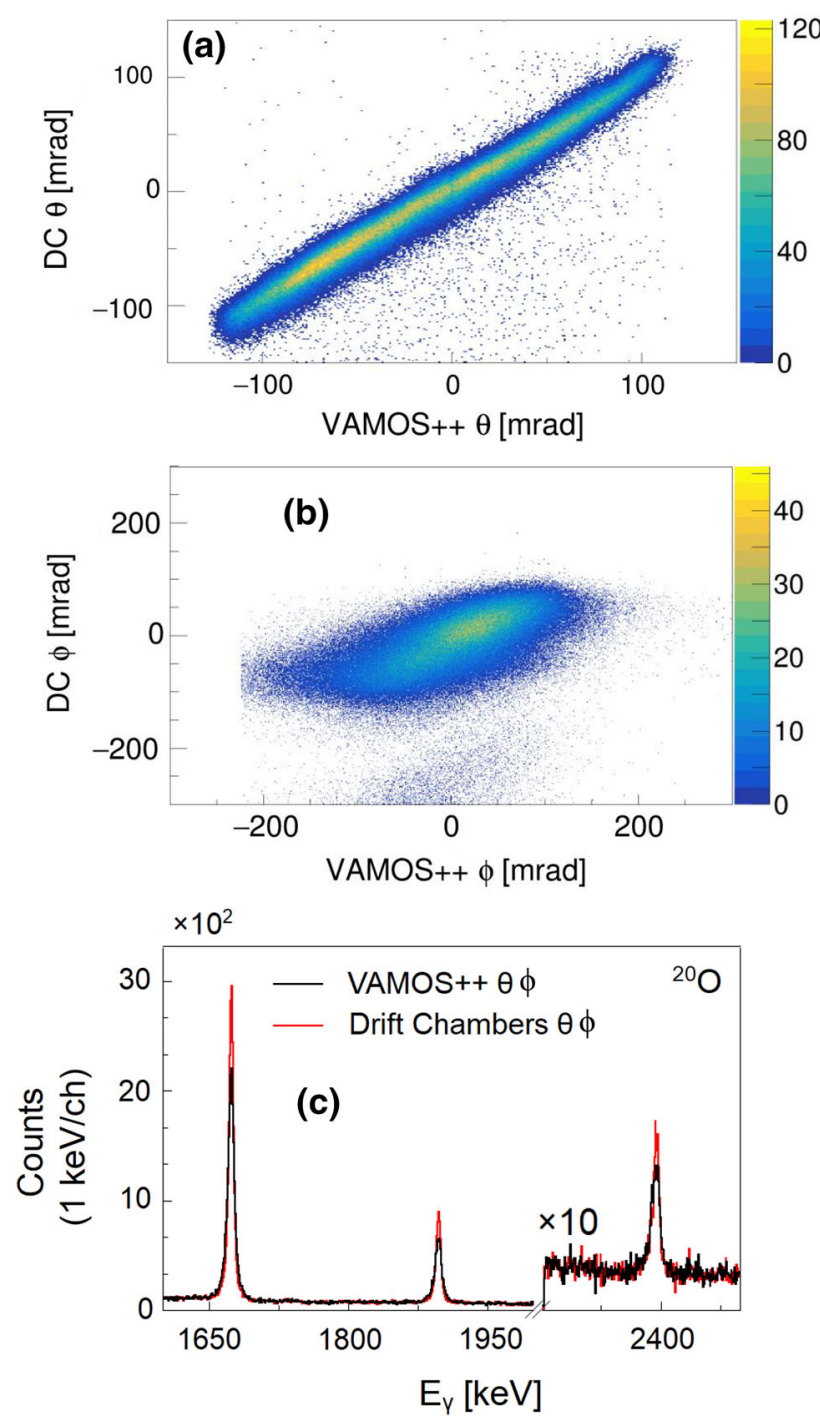

Fig. 3 Panel a, b: drift chambers (DCs) vs. VAMOS++ reconstructed $\theta$ and $\phi$ angles of the recoiling ions, respectively. Panel c: comparison between Doppler-shift corrected ${ }^{20} \mathrm{O} \gamma$-ray spectra measured in AGATA, based on the reconstructed $\theta$ and $\phi$ angles from the VAMOS++ focal-plane detectors only (black), or on the use of the entrance drift chambers (red). The FWHM of the peaks improves by a factor of 1.4 for $\gamma$-ray energies around $1.6-1.9 \mathrm{MeV}$

(b). Panel (c) displays a $\gamma$ spectrum of ${ }^{20} \mathrm{O}$, as measured in AGATA, obtained after applying a Doppler-shift correction in which the ion direction reconstruction was based on the VAMOS++ focal-plane detectors only (black line), or on the use of the entrance drift chambers (red line). We remark that a similar improvement of the Doppler reconstruction was also discussed in Ref. [28], where a Multi-wire chamber was used for heavy and slow ions detection. In the following, the more accurate angles from the entrance DCs will be used for the ion direction entering the Doppler-shift correction, rather than the focal-plane reconstructed ones (see Sect. 3.2.1).

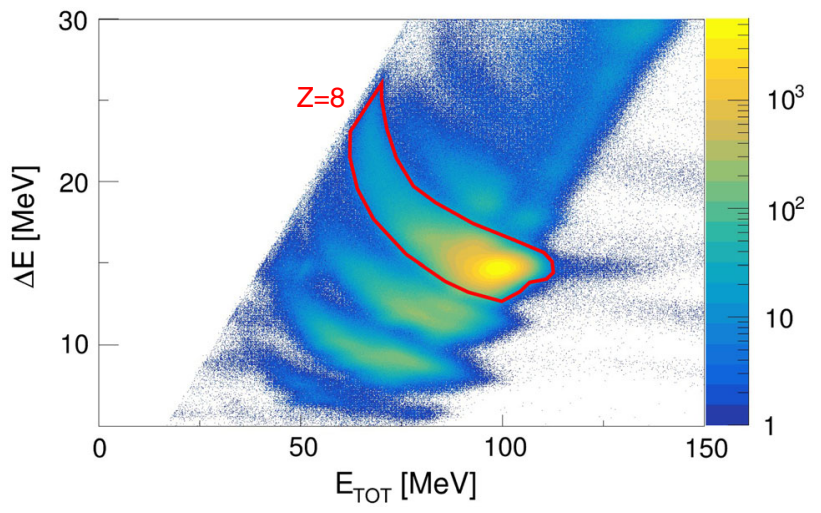

Fig. $4 \Delta \mathrm{E}^{\mathrm{v}}$. $\mathrm{E}_{T O T}$ two-dimensional histogram, where $\triangle \mathrm{E}$ and $\mathrm{E}_{T O T}$ are the energy loss and total ion energies measured by the ionization chambers and focal-plane plastic detectors of the VAMOS++ magnetic spectrometer, in the ${ }^{18} \mathrm{O}(126 \mathrm{MeV})+{ }^{181} \mathrm{Ta}$ experiment. As an example, the region corresponding to $\mathrm{Z}=8$ is encircled by a red line [25]

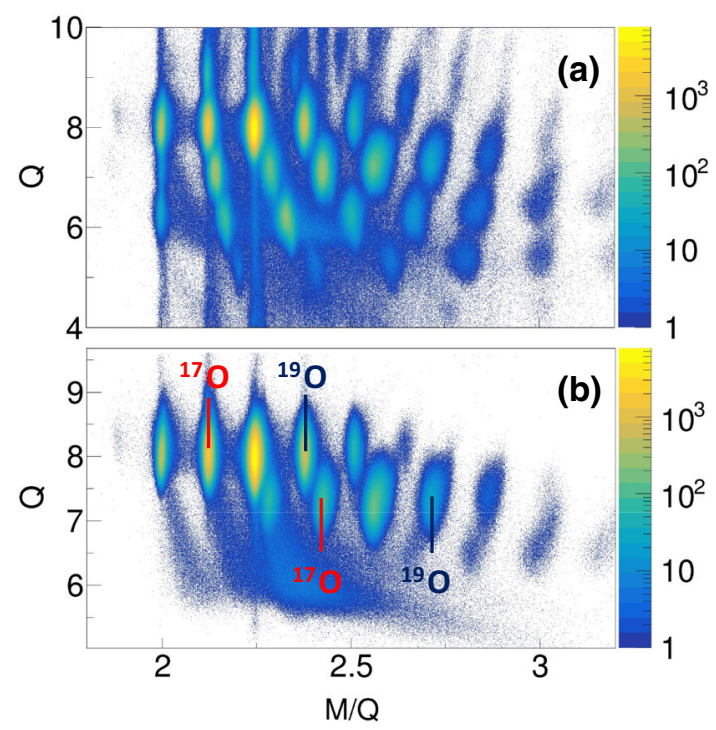

Fig. 5 Plot of the ion charge Q vs. M/Q for all ions (a) and for the selection of oxygen $(Z=8)$ isotopes $(\mathbf{b})$, with labels pointing to ${ }^{17} \mathrm{O}$ and ${ }^{19} \mathrm{O}$ for each charge state, separately. Data refer to the ${ }^{18} \mathrm{O}(126 \mathrm{MeV})+$ ${ }^{181}$ Ta experiment [25]

The ion velocity in VAMOS,$++ v_{V}$, the ion mass $M$ and charge state $Q$ were obtained by employing the standard VAMOS++ analysis procedure [19,20,31]. The ion atomic number $Z$ was determined from the correlation between the energy loss $\triangle \mathrm{E}$ and the total energy $\mathrm{E}_{T O T}$, as shown in Fig. 4. Figure 5 shows the plot of the product-charge state $Q$ versus $M$ for all $Z$ (panel a), and for $Z=8$ isotopes (panel $\mathrm{b}$ ).

Two corrections were introduced to the standard VAMOS++ identification method, in order to improve the determination of ion mass and trajectory. First, a check was done on the stability of the reconstructed masses in the course of the measurement. Panel (a) of Fig. 6 shows the evolution of the reconstructed ${ }^{18} \mathrm{O}$ ion mass, as a function of the experiment 
duration time. A clear drift is visible, in phase with the drift observed for the time signal of the PARIS scintillators, being both taken with respect to the cyclotron radiofrequency (RF), which was slightly fluctuating (panel b). It follows that a correction to the drift in the ion time-of-flight $\mathrm{T}$ (from the target to the focal plane plastic detector), on which the mass reconstruction is based, can be extracted from the PARIS time vs. RF drift. As shown in Fig. 6c, this leads to a significantly improved stability of the reconstructed masses and to an overall improved mass resolution (panel d).

Second, for what concerns the ion trajectory reconstruction, a significant improvement in the $\gamma$-ray Doppler-shift correction is obtained by considering a finite-size instead of a point-like beam spot. The position of the beam on target can in fact be deduced by considering the ion track direction, as measured by the two pairs of drift chambers at the entrance of VAMOS++. Considering the geometry of the setup (i.e., target-detector distances) and this ionreconstructed direction, a Gaussian-like beam-on-target distribution was obtained, with $\sigma_{x}=0.5 \mathrm{~mm}$ and $\sigma_{y}=0.4 \mathrm{~mm}$. In the data analysis, only ion trajectories originating within a $4 \mathrm{~mm}$ distance from the beam-spot center were considered, leading to the rejection of wrongly reconstructed trajectories and improving the $\gamma$-ray Doppler-shift correction, in comparison with a point-like beam-spot assumption.

\subsection{Offline processing of the AGATA data}

The most accurate treatment of the information from the AGATA $\gamma$-tracking array is crucial for a precise $\gamma$-ray lineshape analysis (see Sect. 4.5). A data replay was therefore performed offline, using the NARVAL data-acquisition system emulator [32,33]: all the files containing the electronic traces from each AGATA crystal were processed, the Pulse Shape Analysis (PSA) and the matching of the data from different crystals were repeated, as well as the merging of the events from AGATA and ancillary detectors. The energy and direction of the interacting $\gamma$ ray in AGATA were reconstructed by the combined use of the PSA [33,34] and of the Orsay Forward Tracking (OFT) algorithm [35], which allow to reach a position resolution of the order of $4 \mathrm{~mm}$ FWHM. During the offline data replay, crosstalk and neutron-damage corrections were applied, following the procedures described in Refs. [36,37]. Figure 7 illustrates the impact of the neutrondamage corrections on the 36 segment energy spectra of crystal 10B of AGATA, which results in a clear improvement in the peak symmetry and corresponding energy resolution.

The offline data replay was also needed for statistics recovery from missing or broken crystal segments, timestamp alignment (the timestamp frequency is $100 \mathrm{MHz}$ ) and energy-calibration improvements. In particular, by setting stringent gates on the timestamp difference between AGATA and VAMOS++, an improvement of the peak-to-background

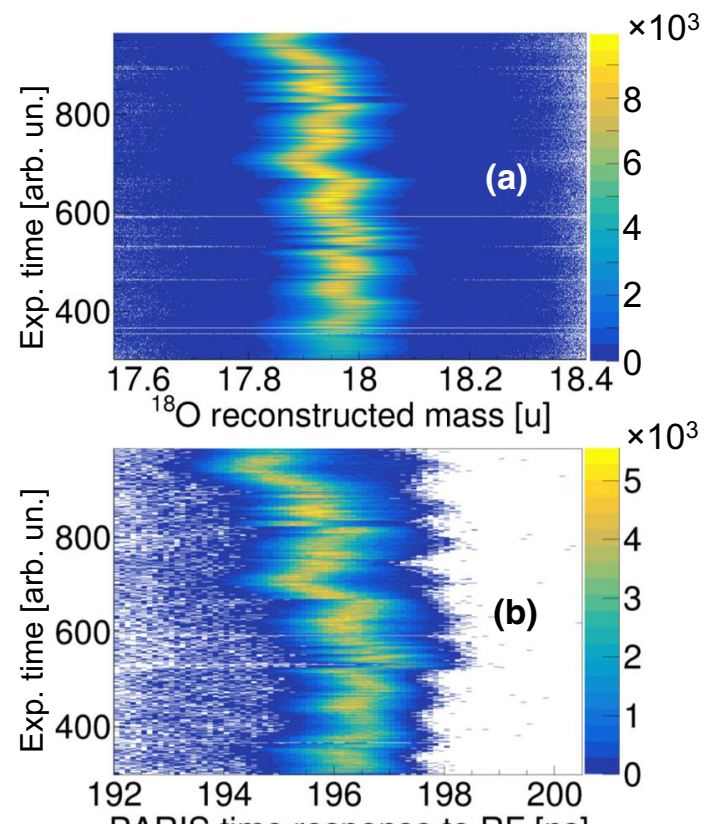

PARIS time response to RF [ns]
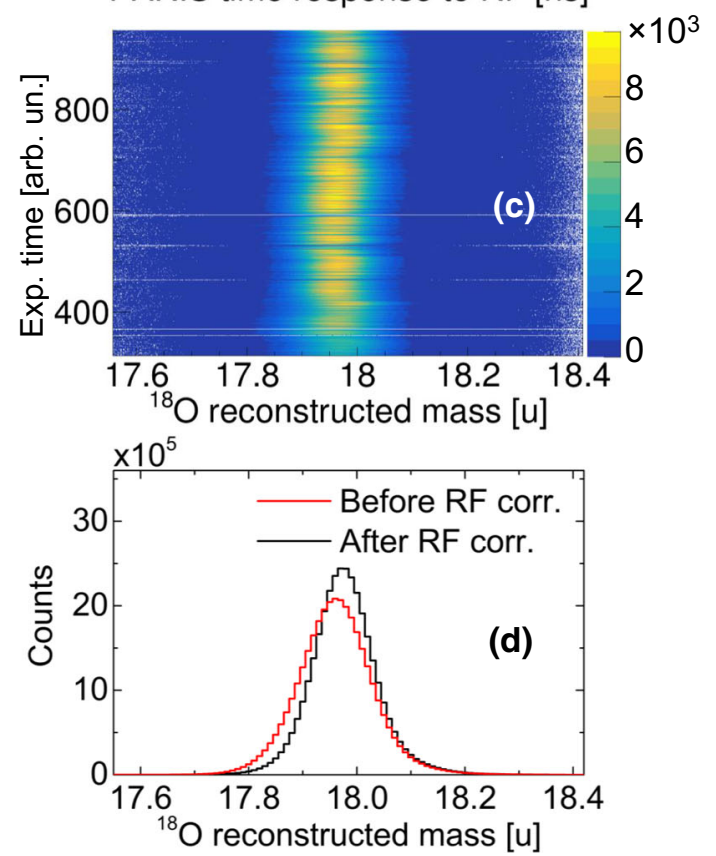

Fig. 6 a Reconstructed ${ }^{18} \mathrm{O}$ mass, as a function of the experiment duration time. b PARIS time signal with respect to the cyclotron RF. c Reconstructed ${ }^{18} \mathrm{O}$ mass after the RF drift correction, estimated from the PARIS time drift with respect to the RF (panel b). d ${ }^{18} \mathrm{O}$ reconstructed mass before (red) and after (black) correction for the RF drift. The FWHM improves by a factor of 1.2

ratio of a factor $>2$ was achieved, with a loss of counts in the photopeak less than $4 \%$.

An accurate energy calibration was then applied to the core signals of the AGATA detectors, using $\gamma$-ray lines from ${ }^{152} \mathrm{Eu}$, below $1.5 \mathrm{MeV}$, and background radiation in the $2-3$ $\mathrm{MeV}$ energy region (e.g., $\gamma$-rays from ${ }^{208} \mathrm{~Pb}$ natural back- 


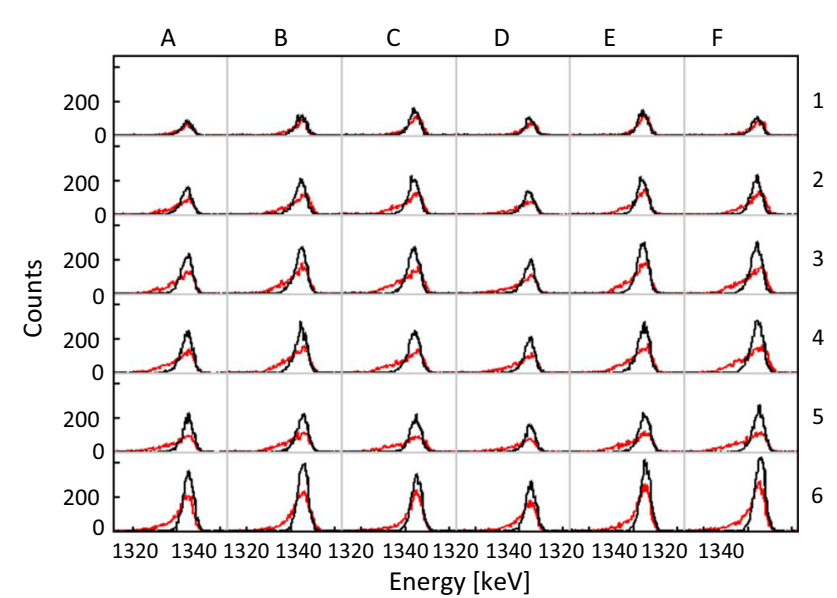

Fig. 7 Examples of energy lineshapes for the $1332-\mathrm{keV}{ }^{60} \mathrm{Co} \gamma$-ray from the 36 segments of crystal 10B of AGATA, before (red) and after (black) the neutron-damage correction [37]. The source data refers to a calibration run performed for the ${ }^{18} \mathrm{O}+{ }^{181} \mathrm{Ta}$ experiment. See also Fig. 22 for segments label code

ground and from ${ }^{24} \mathrm{Mg}$, produced by $\beta$ decay of ${ }^{24} \mathrm{Na}$, following neutron capture on the NaI crystals of the PARIS array). The energy calibration was improved by forcing the summed energy measured in the crystal segments to be equal to the one measured in the core, for each $\gamma$ ray (the ForceSegmentsToCore option of the AGATA software package was used [38]). After this procedure, some missing energy could be recovered, resulting in a further reduction of the left-side tail of the energy peaks (in addition to the neutron-damage and crosstalk corrections). For one crystal (ID. 42), the core signal was degraded, therefore the energies of the individual segments were summed up, after being individually calibrated.

At the end of the calibration process, discrepancies between tabulated and calibrated energies below $0.2 \mathrm{keV}$ were obtained for most of the detectors, with only 4 detectors having discrepancies around $0.5 \mathrm{keV}$. Figure 8 shows examples of two-dimentional histograms of AGATA energy spectra from calibration sources, before (a) and after (b) the energy-calibration procedure, in the region of the $2754-\mathrm{keV}$ line from ${ }^{24} \mathrm{Mg}$. As shown in panel (c), an overall improvement of a factor of 1.4 is obtained in the FWHM of the 2754$\mathrm{keV}$ line. Possible energy gain drifts with time were also checked using calibration sources, and no appreciable drift was observed.

\subsubsection{Doppler-shift correction}

After merging the AGATA and ancillaries events, via timestamps correlations, the Doppler-shift correction was applied to the $\gamma$-ray energies according to the formula:

$E_{\gamma_{0}}=E_{\gamma} \times \gamma\left(1-\beta \cos \left(\theta_{\text {rel }}\right)\right)$
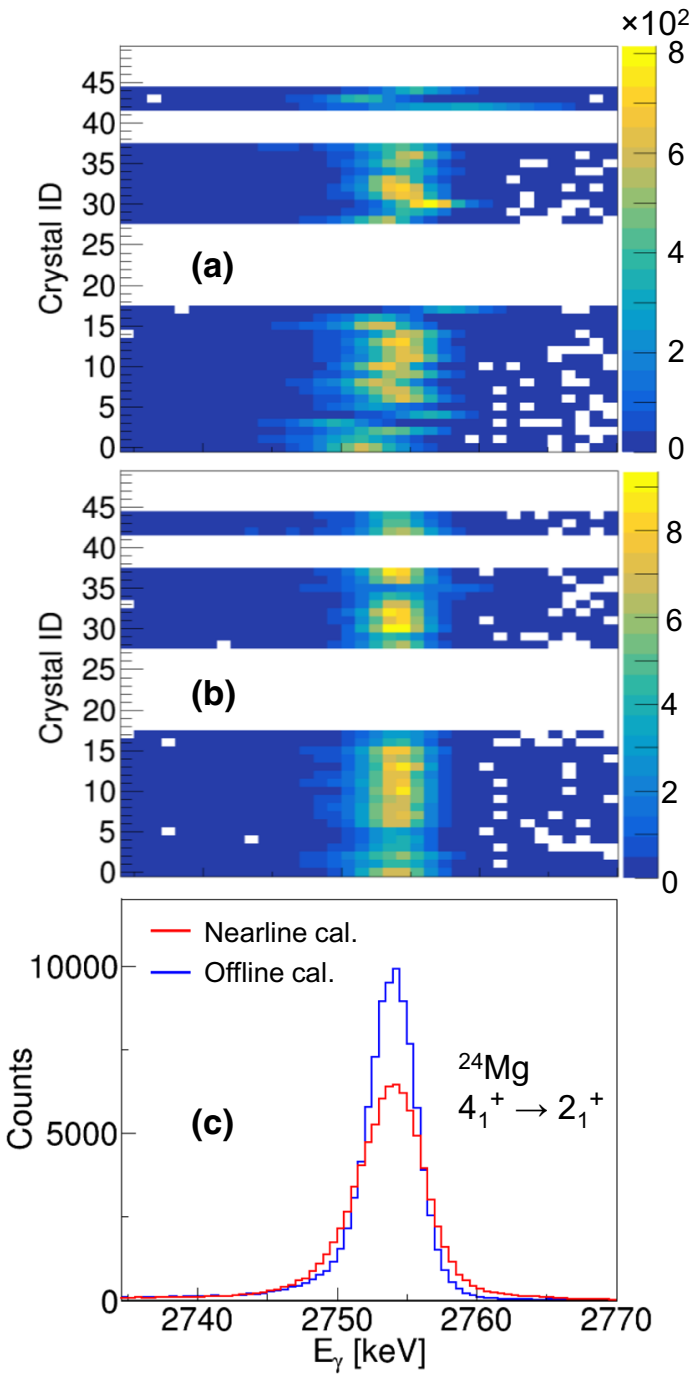

Fig. 8 Examples of two-dimensional histograms showing the AGATA crystal identification number (Crystal ID, y axis) vs. energy spectra from calibration sources, in the region around the 2754-keV line from ${ }^{24} \mathrm{Mg}$, before (a) and after (b) the energy-calibration correction procedure. Panel $\mathbf{c}$ shows the corresponding energy spectra, summed over all AGATA crystals, from which an improvement of a factor of 1.4 is deduced in the FWHM (see text for details)

where $E_{\gamma}$ and $E_{\gamma_{0}}$ are the measured and Doppler-shift corrected $\gamma$-ray energies, respectively. $\theta_{\text {rel }}$ is the angle between the direction of the reaction product (measured in the entrance drift chambers) and the emitted $\gamma$-ray direction, which is calculated by considering the $\gamma$ emission from the target center and the first interaction point in AGATA (see discussion in Sect. 4.5). In Eq. 1, the $\beta$ and $\gamma$ relativistic terms are calculated, event by event, by using the velocity after the target, $v=v_{V}+\Delta v_{V}$, where $v_{V}$ is the velocity measured in VAMOS++, while $\Delta v_{V}=T /\left(v_{V}^{2} f(\theta, \phi)\right)$ is a velocity correction based on the energy loss in the entrance drift chambers: $T$ is a parameter calculated from the LISE code [39] (assuming the stopping power for oxygen ions, 


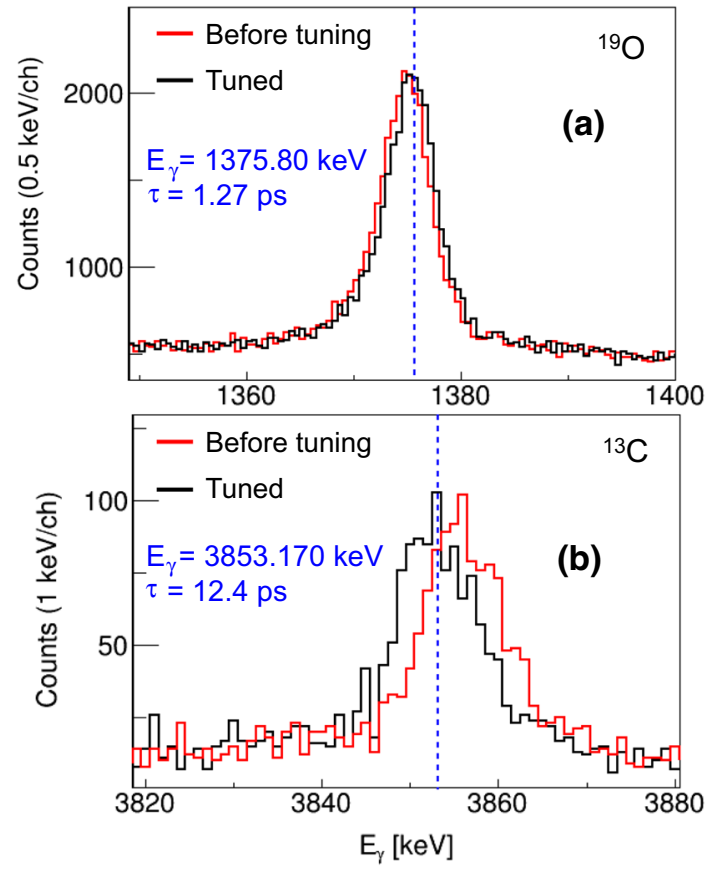

Fig. 9 Effect of the fine-tuning of the ion velocity (after corrections for the energy loss in the entrance DC's detectors) on the Doppler-shift corrected 1375.80(8)-keV and 3853.170(22)-keV $\gamma$-rays of ${ }^{19} \mathrm{O}$ (a) and ${ }^{13} \mathrm{C}(\mathbf{b})$, respectively. The corresponding changes in velocity are $0.4 \%$ and $0.7 \%$. Dashed blue lines indicate the nominal $\gamma$-ray energies $[40,41]$

i.e., projectile-like reaction products with $Z=8$ ), while $f(\theta, \phi)$ is a function whose value is proportional to the ion-path length in the entrance DCs. An additional fine tuning of the after-target velocity (i.e., of the $T$ parameter) is performed by considering the Doppler-shift corrected $\gamma$-ray energies for known transitions de-exciting long-lived states (with $\tau>1 \mathrm{ps,} \mathrm{i.e.,} \mathrm{decaying} \mathrm{after} \mathrm{the} \mathrm{target),} \mathrm{and} \mathrm{by}$ comparing them to their nominal energies, reported in literature. The $T$ parameter is established for a given $Z$ of the product, by matching those energies, as illustrated in Fig. 9 for the $1375.80(8)-\mathrm{keV} \gamma$ ray in ${ }^{19} \mathrm{O}$ (a) and the $3853.170(22)-\mathrm{keV} \gamma$ ray in ${ }^{13} \mathrm{C}(\mathbf{b})$. The corresponding changes in velocity are $0.4 \%$ and $0.7 \%$ for oxygen and carbon ions, respectively.

Following the procedure described in the previous sections, ion-gated $\gamma$-ray spectra measured in AGATA were constructed and Doppler-shift corrected using the ion velocity after the target. Figures 10 and 11 show, as examples, portions of Doppler-shift corrected $\gamma$-ray spectra of ${ }^{17} \mathrm{O}$ and ${ }^{19} \mathrm{O}$ and corresponding level schemes. Transitions of 2184- and 3843-keV energy in ${ }^{17} \mathrm{O}$, and of 2371- and 2779$\mathrm{keV}$ energy in ${ }^{19} \mathrm{O}$ will be later considered for lifetime analyses.
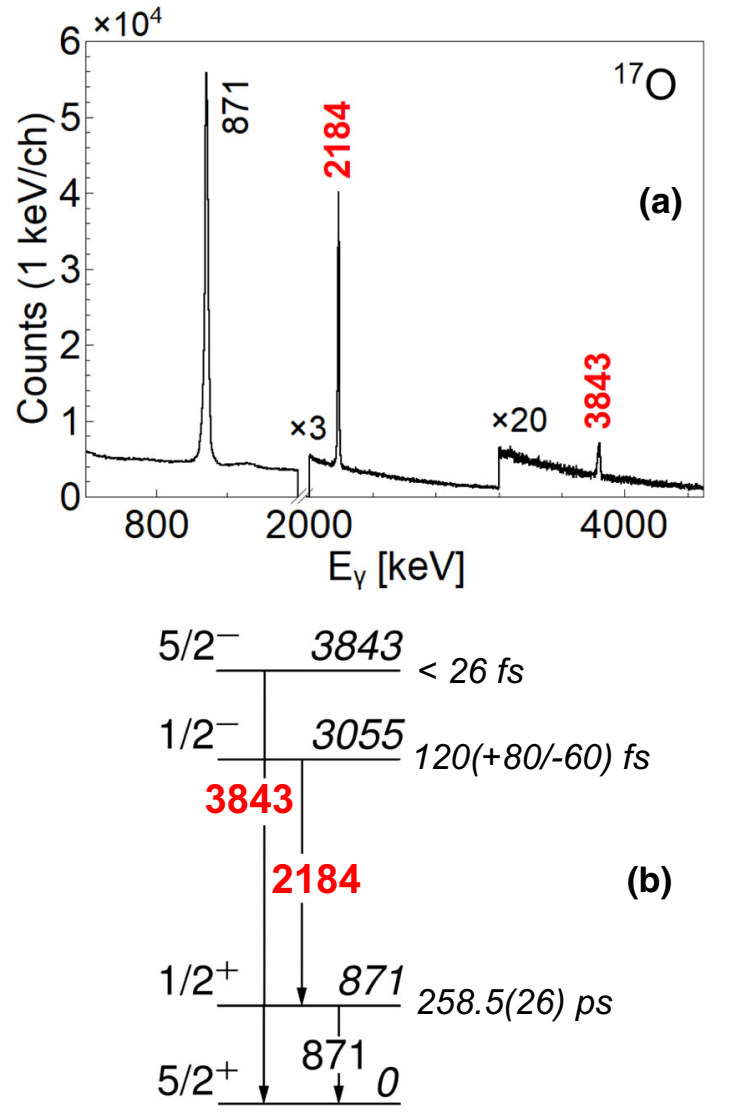

Fig. 10 Panel a: ${ }^{17} \mathrm{O} \gamma$-ray spectrum measured in AGATA, Dopplershift corrected considering the ion velocity after the target. Panel b: corresponding level scheme, with energies and state lifetimes taken from literature [41]. Transitions marked in red are considered in the lifetime analysis discussed in this work

\section{Monte Carlo simulations for lifetime analysis}

The present section describes the Monte Carlo simulation on which the lifetime analysis is based. Nuclear states populated in low-energy binary heavy-ion reactions are considered, with decay time in the range of tens-to-hundreds femtoseconds, i.e., of the target-crossing time $\left(T_{\text {cross }} \sim 130 \mathrm{fs}\right)$. The procedure consists of three major steps: i) a preliminary Monte Carlo calculation to reconstruct the Total Kinetic Energy Loss (TKEL) distribution, for the population of a given nuclear state of the projectile-like product (Sect. 4.1), ii) the simulation of the AGATA Doppler-shift corrected $\gamma$ ray spectrum, which is based on the projectile-like velocity calculated from the reconstructed TKEL (Sect. 4.2), and iii) the two-dimensional $\chi^{2}$ minimization procedure, in lifetime-transition energy coordinates, based on the comparison between simulated and experimental $\gamma$-transition lineshapes (Sect. 4.3). 


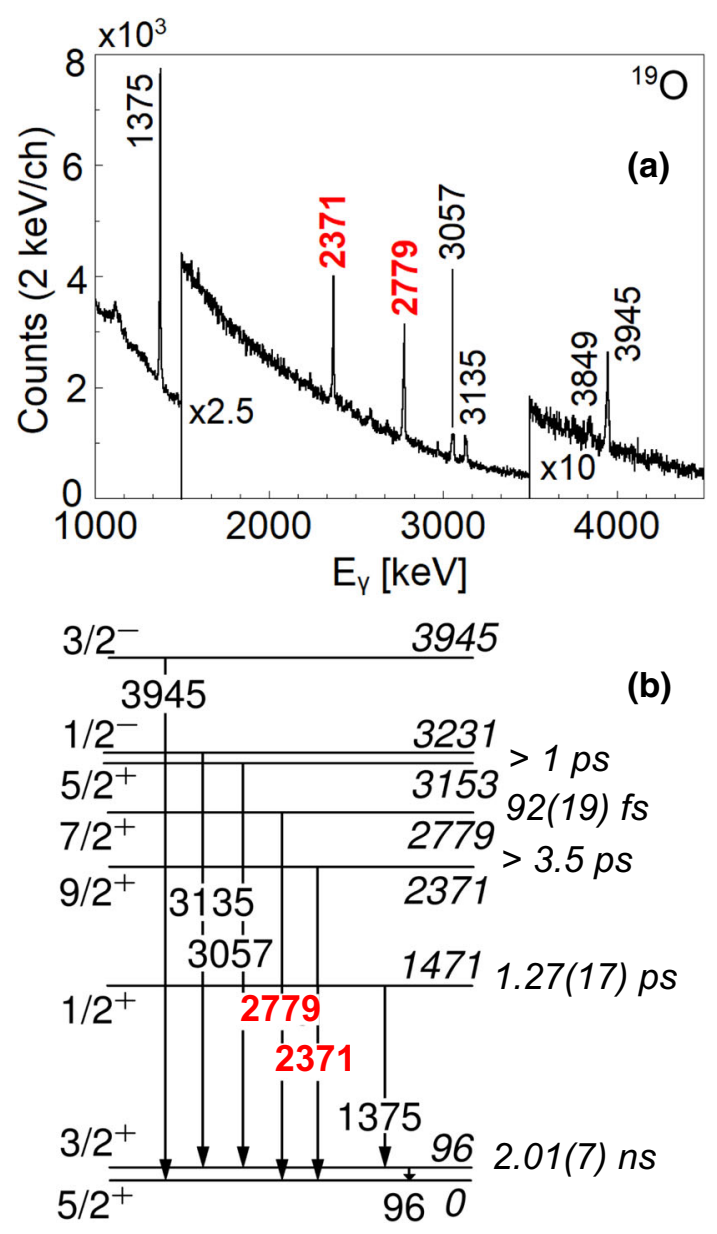

Fig. 11 Panel a: ${ }^{19} \mathrm{O} \gamma$-ray spectrum measured in AGATA, Dopplershift corrected considering the ion velocity after the target. Panel $\mathbf{b}$ : corresponding level scheme, with energies and state lifetimes taken from literature [41]. Transitions marked in red are considered in the lifetime analysis discussed in this work

\subsection{Reconstruction of the initial velocity distribution}

The key point of the entire procedure is the determination of the velocity vector of the projectile-like product at the reaction instant, for a given excited state population. In the case of low-energy binary heavy-ion reactions, the velocity distribution of the reaction product includes contributions from both direct (quasi-elastic) and more dissipative processes, which lead to the appearance of broad structures at lower velocities [4-9]. This is demonstrated in Fig. 12, in the case of ${ }^{19} \mathrm{O}$. Panel (a) shows the matrix $\mathrm{E}_{\gamma}$ vs. measured ion velocity for AGATA Doppler-shift corrected $\gamma$-rays. Transitions of energies 1375, 2371 and $2779 \mathrm{keV}$ are clearly visible, depopulating excited states at 1471, 2371 and $2779 \mathrm{keV}$ (see level scheme in Fig. 11). Panels (b)-(d) show velocity distributions gated on each transition. Only in the case of the $1 / 2^{+}$ state at $1471 \mathrm{keV}$ (see panel b), a Gaussian-like velocity distribution is observed, while decays from other states display

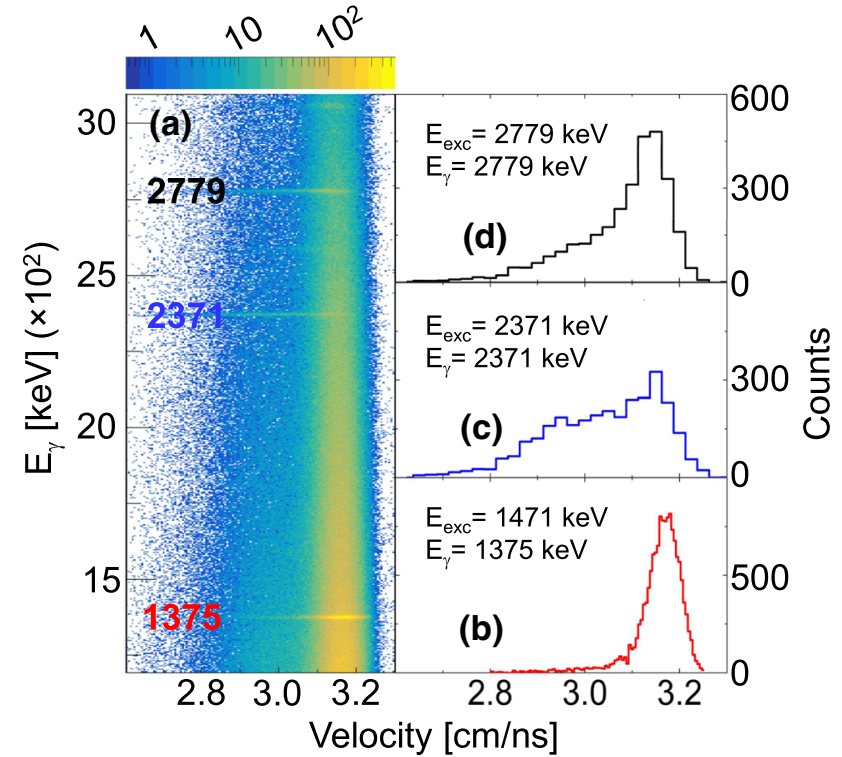

Fig. 12 Two-dimensional plot of AGATA Doppler-shift corrected $\gamma$ ray energies vs. ions velocities, as measured in VAMOS++, in the case of ${ }^{19} \mathrm{O}$ (a). Velocity distributions obtained by gating on the 1375- (b), 2371- (c) and 2779-keV (d) $\gamma$ rays depopulating the 1471-, 2371- and 2779-keV excited states of ${ }^{19} \mathrm{O}$

significant contributions, at lower velocities, from dissipative processes.

Since dissipative contributions cannot be reliably calculated, a Monte Carlo procedure had to be developed to reconstruct the TKEL distribution, where TKEL is defined as the difference between the total kinetic energy before and after the collision. This calculation is performed prior to the simulation of the $\gamma$-ray emission (which is the subsequent step discussed in Sect. 4.2) and it is based on a recursive subtraction, from the measured velocity distribution, of the velocity components associated with consecutive bins of TKEL.

As illustrated in the flow diagram of Fig. 13, in the first iteration the velocity component associated with the direct population of the state of interest, at energy $\mathrm{E}_{0}^{*}$, is calculated from the two-body reaction kinematics. The calculation is done looping over 100 target layers into which the target was divided: in each layer the beam-energy degradation is about $0.1 \mathrm{MeV}$, resulting in an energy loss of $\sim 10 \mathrm{MeV}$ in the full target. The reaction is assumed to occur at random over the full target thickness, being the deep-inelastic reaction cross section (at energies $\sim 60 \%$ above the Coulomb barrier) rather constant for beam energy variations of the order of $\sim 10 \%$, as in the present case. The slowing down of the beam and projectile-like reaction product and their energy and angular straggling in the target are also considered, following the prescription of the LISE code [39]. For each event, a reactionproduct scattering angle is randomly selected from the measured angular distribution in VAMOS++. This automatically takes care of the acceptance of the magnetic spectrometer 

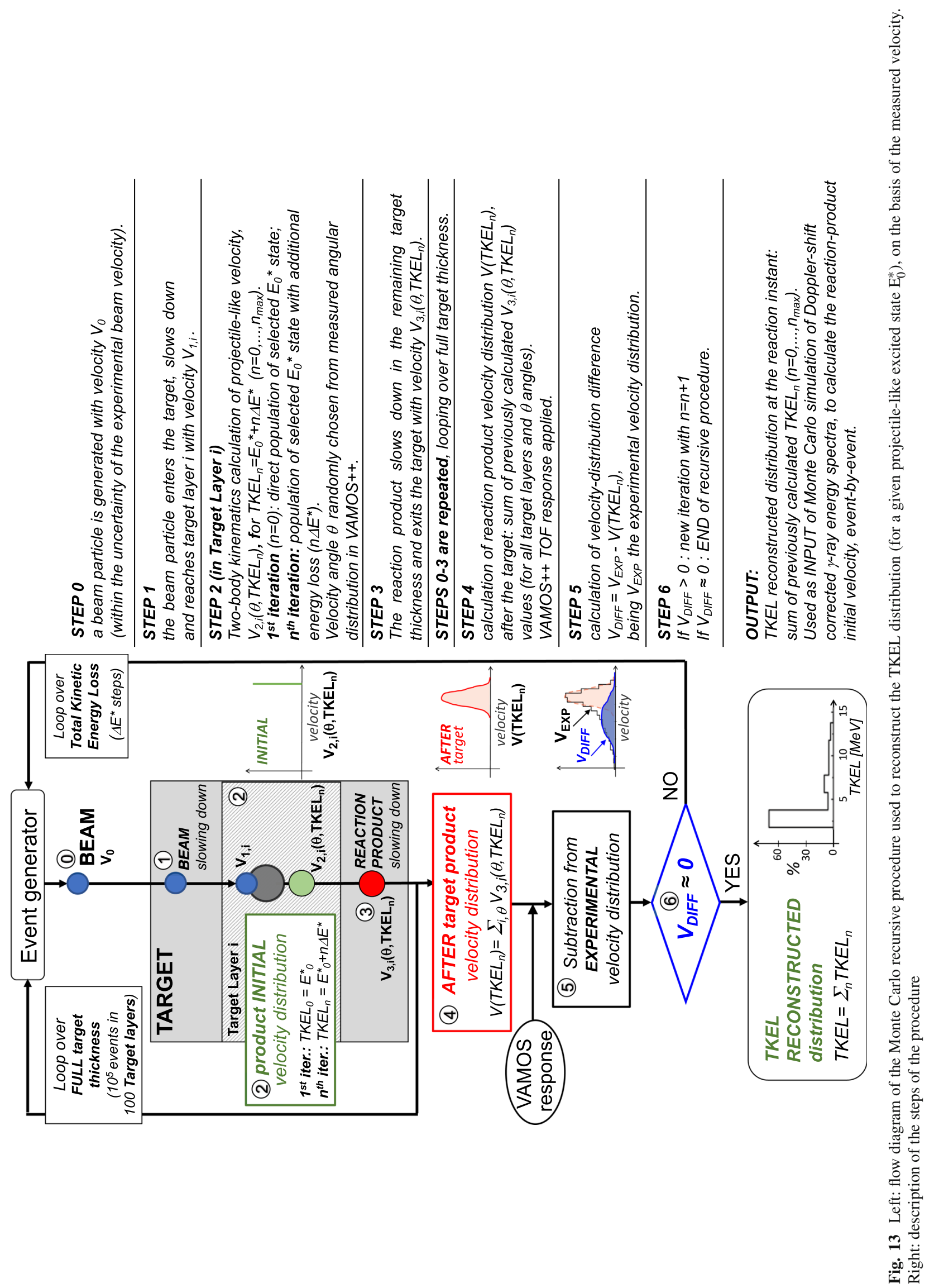
[20]. The velocity distribution, after the target, is then folded with the VAMOS++ response which is dominated by the time-of-flight (TOF) uncertainty (with $\sigma=1 \mathrm{~ns}$ ). The final simulated velocity distribution is then subtracted from the experimental one, after proper normalization.

In the next iteration, the second component of the projectile-like velocity distribution, associated with a TKEL increase by $\Delta \mathrm{E}^{*}$, is calculated following a procedure similar to the first iteration, and the corresponding final velocity distribution, after the target, is also corrected for the VAMOS++ TOF response and subtracted from the remaining measured velocity distribution. In the present analysis, a number $n_{\text {max }}$ of 10 iterations was considered (with $\Delta \mathrm{E}^{*}=2$ $\mathrm{MeV}$, consistently with the VAMOS++ energy resolution), in order to fully reproduce the experimental velocity distribution, although a few iterations were found sufficient in all treated cases.

As a result of this preliminary Monte Carlo calculation, the TKEL distribution associated with the population of a given $\mathrm{E}_{0}^{*}$ state of the projectile-like product is reconstructed (see bottom of Fig. 13). Such a TKEL distribution will be used in the main simulation of the AGATA Doppler-shift corrected $\gamma$-ray spectrum to calculate, event-by-event, the projectilelike product velocity vector, at the reaction instant, from the reaction kinematics (see Sect. 4.2).

Figure 14 displays, as examples, the velocity distributions for the ${ }^{19} \mathrm{O}$ product excited to the $1471-\mathrm{keV}$ (a) and 2779-keV (b) states, and the corresponding simulated distributions. The measured (black histogram) and simulated (red line) velocity distributions have Gaussian-like shapes in the case of the 1471-keV state (panel a), which is characteristic for an exclusive direct population process. In contrast, the velocity distribution measured for the $2779-\mathrm{keV}$ state (panel b) has a complex structure which is well described by a velocity profile with two separate contributions, associated with direct (red dashed) and dissipative (blue dashed) processes, respectively. In both panels, the yellow solid line displays the unfolded total initial velocity distribution (i.e., summed over the full target thickness), while the green line represents the contributions from the first 10 target layers. In each panel, the inset gives the reconstructed TKEL distribution for the corresponding state population (in $2 \mathrm{MeV}$ bins).

As mentioned above, an important ingredient of the simulation is the stopping-power parameterization, which was taken from Ziegler et al. [42,43]. We evaluated the influence of this choice for our reaction by varying up to $20 \%$ the prescribed value, in the case of a uniquely determined reaction kinematics (i.e., the direct population of the $1471-\mathrm{keV}$ state in ${ }^{19} \mathrm{O}$ ). As shown in Fig. 15, the simulated final velocity distribution (red band) reproduces the measured velocity profile within a $1 \sigma$ uncertainty for stopping-power variation $<5 \%$. We also compared the simulated velocity distributions extracted using the Ziegler et al. parametrization with differ-

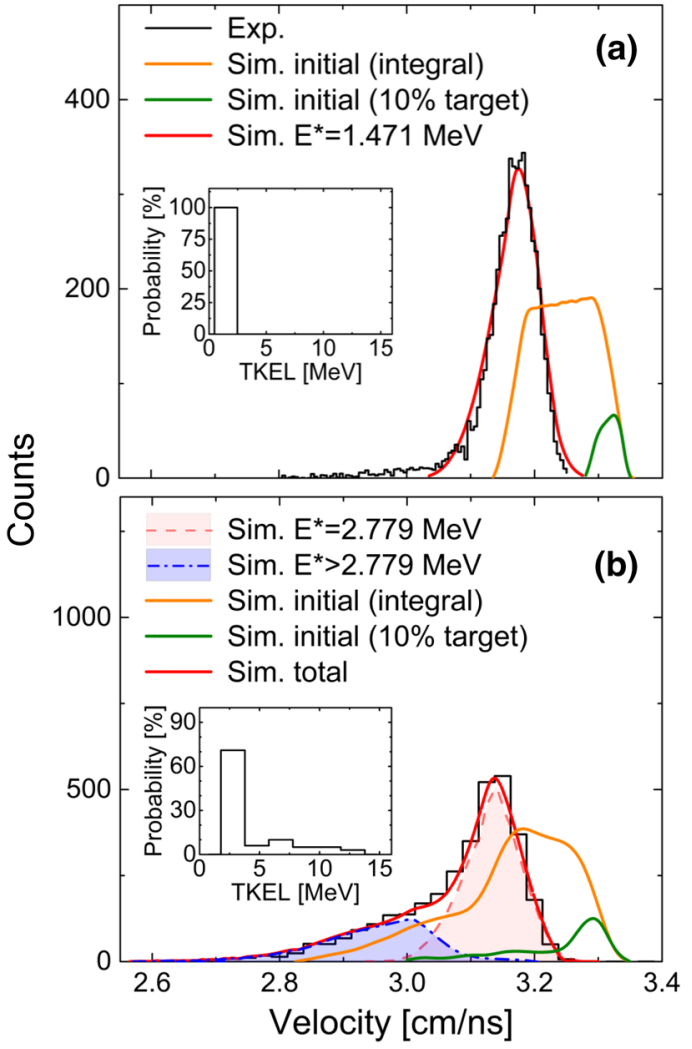

Fig. 14 Panel a: measured (black histogram) and simulated (red solid line) velocity distributions for the de-excitation of the 1471-keV state of ${ }^{19} \mathrm{O}$. The unfolded total initial velocity distribution is shown by the yellow line, while the green line displays the contribution from the first 10 target layers. Panel $\mathbf{b}$ : Same as in panel a, for the de-excitation of the $2779-\mathrm{keV}$ state of ${ }^{19} \mathrm{O}$. Red and blue distributions correspond to simulated direct (quasi-elastic) and dissipative components. The inset, in both panels, gives the reconstructed TKEL distribution (with 2 $\mathrm{MeV} / \mathrm{bin}$ ) for the corresponding state population (see Fig. 13 and text for details)

ent stopping-power laws, such as the one used by the code ATIMA [44], which is usually considered for higher energies. Differences in energy losses were of the order of 2-3\%, resulting in negligible effects in the subsequent analysis.

\subsection{Simulation of AGATA Doppler-shift corrected $\gamma$-ray spectra}

Simulation calculations of an AGATA Doppler-shift corrected $\gamma$-ray lineshape, for a transition depopulating a selected state, were performed in a two-step process.

In the first step, the $\gamma$ events are prepared with a Monte Carlo procedure (with typical number of events of the order of $4 \times 10^{6}$ ), following a flow diagram similar to the one presented in Fig. 13. For each event, after the beam particle reaches the target layer in which the reaction occurs, the velocity of the projectile-like product, at the reaction instant, is obtained from a two-body kinematics calculation assuming 


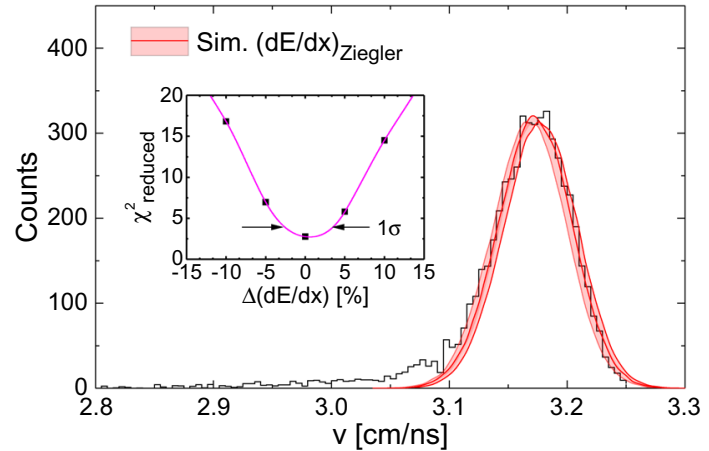

Fig. 15 Measured velocity distribution for the de-excitation of the $1471-\mathrm{keV}$ state in ${ }^{19} \mathrm{O}$. The red-shaded band is obtained by varying by $5 \%$ the stopping-power parametrization of Ziegler et al. [42]. Inset: reduced $\chi^{2}$ curve for Monte Carlo simulations using different $\Delta(\mathrm{dE} / \mathrm{dx})$ variation (in \%) of the Ziegler stopping-power parametrization, pointing to a $1 \sigma$ uncertainty $<5 \%$

a TKEL value randomly chosen from the previously reconstructed TKEL distribution for the specific populated state (see Sect. 4.1). For each event, the velocity direction is again randomly selected within the angular distribution measured in VAMOS++. A Doppler-shifted $\gamma$ ray, emitted from the projectile-like product slowing down in the remaining target thickness, is simulated assuming a given $\gamma$-transition energy and a decay time randomly chosen on the basis of the excitedstate lifetime.

In the second step, the $\gamma$-ray events generated by the Monte Carlo procedure discussed above (each event containing information on $\gamma$ energy, $\gamma$ direction and the ion-velocity vector, after the target) are passed to the AGATA simulation package [45], which provides, as an output, the $\gamma$-ray energy deposited in the AGATA crystals. In the calculations, the AGATA-simulation code considers the actual configuration of the array in the measurement (i.e., 31 crystals with the corresponding geometry of the ${ }^{18} \mathrm{O}(126 \mathrm{MeV})+{ }^{181} \mathrm{Ta}$ experiment [25]). Figure 16 shows a comparison between the experimental and simulated $\gamma$-ray interaction positions projected on the $x-y$ plane of AGATA, and associated with the first interaction point in the detector crystals. The strong similarity between the two distributions gives support to the quality of the AGATA-simulation code.

The simulated $\gamma$-ray data are subsequently analyzed with the AGATA OFT algorithm [35], following the same procedure applied to the experimental data. This allows to obtain the $\gamma$-ray energy and the relative angle between the $\gamma$-ray direction (reconstructed with the AGATA simulation package [45]) and the ion velocity vector, at the decay point (resulting from the Monte Carlo simulation procedure performed in the first step, as discussed above). The $\gamma$-ray Doppler-shift correction is then performed. At this step, corrections are included to take into account the actual experimental energy

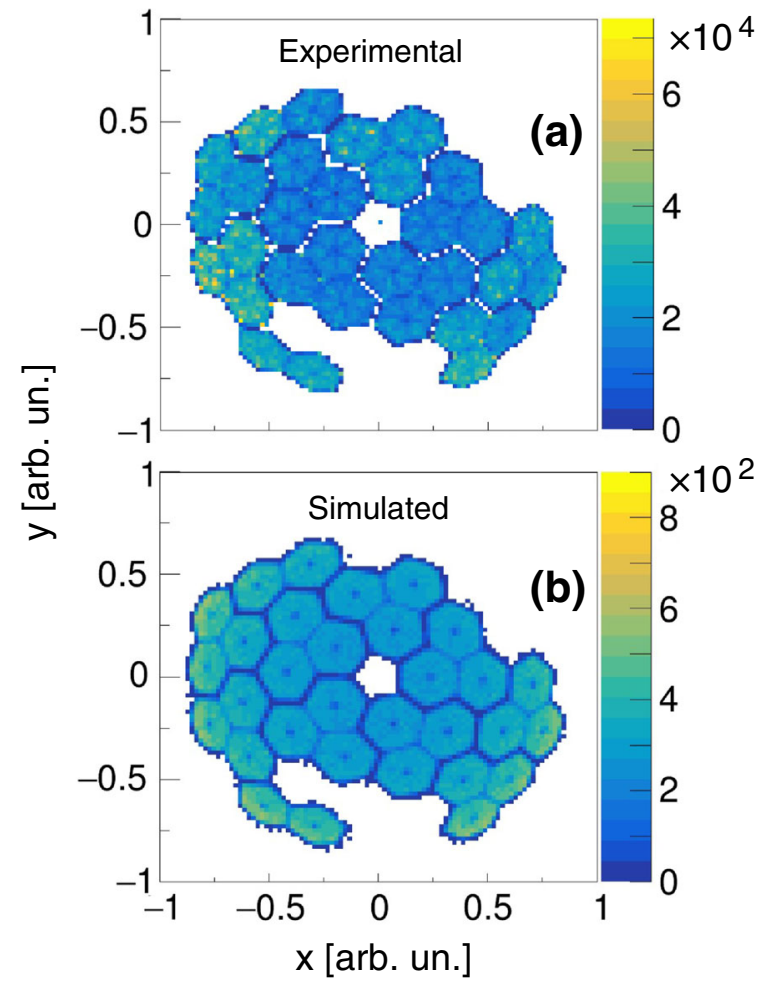

Fig. 16 Experimental view of the $x-y$ plane projection of the $\gamma$-ray interaction positions of the AGATA crystals (a) compared to the simulated ones $(\mathbf{b})$, in the case of the present ${ }^{18} \mathrm{O}(126 \mathrm{MeV})+{ }^{181} \mathrm{Ta}$ experiment [25]. The axes report the $x$ and $y$ positions of the first interaction point divided by the radius of the AGATA array. The $(x, y)=(0,0)$ coordinate center corresponds to the VAMOS++ axis

resolution and the counting statistics of the AGATA detectors.

\section{$4.3 \chi^{2}$ analysis of Doppler-broadened lineshapes for lifetimes determination}

The evaluation of nuclear-state lifetimes in the time range of hundreds femtoseconds requires a detailed study of Dopplerbroadened $\gamma$-ray lineshapes, as a function of the relative angle $\theta_{\text {rel }}$ between the moving-source and the emitted $\gamma$ ray directions. Figure 17 shows examples of simulated lineshapes for the $2.779-\mathrm{MeV} \gamma$ ray of ${ }^{19} \mathrm{O}$, over the full continuous-angle range $\theta_{\text {rel }}=0^{\circ}-180^{\circ}$. In the ${ }^{18} \mathrm{O}+{ }^{181} \mathrm{Ta}$ reaction (sketched in panel (a)), such a transition de-excites the $2.779-\mathrm{MeV}$ state populated by both direct and dissipative processes, as shown in Fig. 14b. In the simulations, three lifetime values are considered, i.e., $\tau=20,100$ and 2000 fs (panels (b), (c) and (d), respectively). In the short lifetime cases, a significant distortion of the overall lineshape is observed, which is at the basis of the nuclear-state lifetime evaluation. We underline that a lifetime-analysis procedure based on such a continuous-angle distribution, which is possible with $\gamma$-tracking arrays, acquires a significantly 

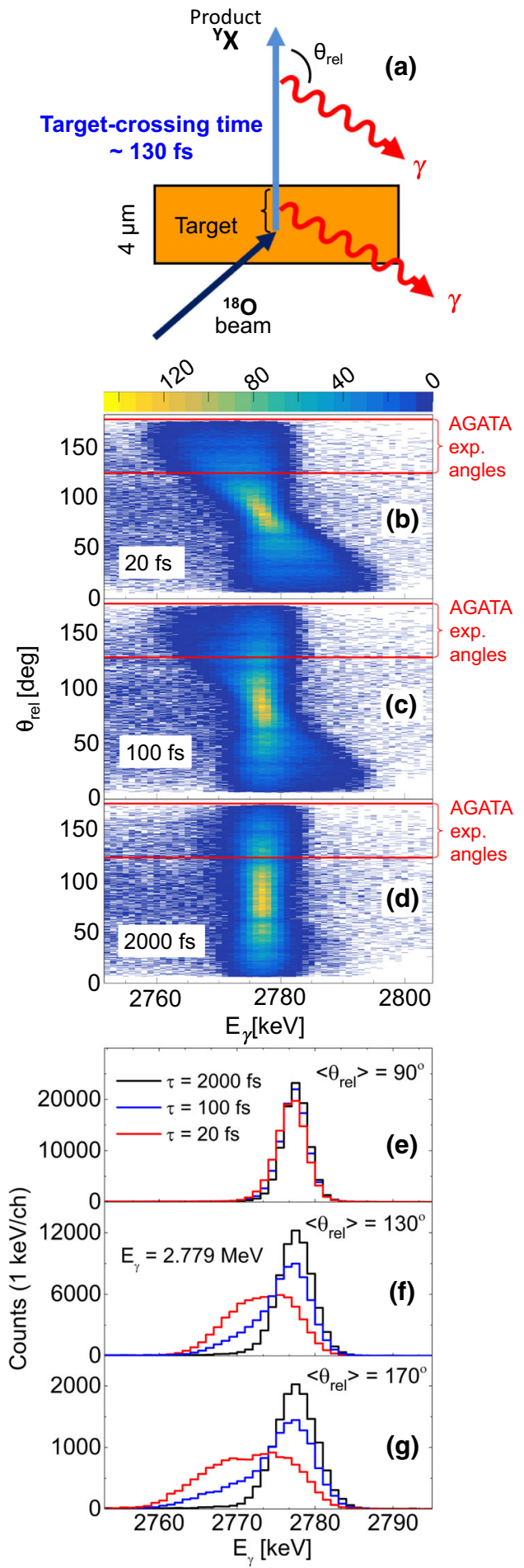

Fig. 17 Panel a: illustration of the interaction process of an ${ }^{18} \mathrm{O} 126-$ $\mathrm{MeV}$ beam in a $4-\mu \mathrm{m}$ thick ${ }^{181} \mathrm{Ta}$ target, resulting in a ${ }^{Y} \mathrm{X}$ reaction product which de-excites by $\gamma$-ray emission. $\theta_{\text {rel }}$ is the angle between the reaction product velocity vector, after the target (as measured in the VAMOS++ spectrometer), and the emitted $\gamma$-ray direction. Panels $\mathbf{b}-\mathbf{d}$ : simulated two-dimensional $\left(\mathrm{E}_{\gamma}, \theta_{\text {rel }}\right)$ Doppler-corrected lineshapes, for the $2.779-\mathrm{MeV} \gamma$ ray deexciting the $2.779-\mathrm{MeV}$ state of ${ }^{19} \mathrm{O}$, assuming lifetime values of 20 (b), 100 (c) and 2000 (d) fs. Horizontal lines give the AGATA angular coverage of the GANIL experiment. Panels e-g: projections on the $\gamma$-ray energy axis for $\theta_{\text {rel }}=90^{\circ} \pm 10^{\circ}(\mathbf{c}), 130^{\circ} \pm 10^{\circ}$ (d) and $170^{\circ} \pm 10^{\circ}(\mathbf{e})$ enhanced sensitivity, with respect to experiments done with conventional $\gamma$-ray arrays with detectors placed at discrete angles, relative to the beam axis (see also Fig. 22). This improvement was already pointed out by C. Stahl et al., [46] and C. Michelagnoli et al., [47] for the restricted cases of reactions in which products have well-defined velocity vectors, such as Coulomb-excitation, transfer and fusion reactions. In our work, we broaden the applicability of such a continuousangle technique to reactions with complex structure of product velocity distribution, as in the case of low-energy binary, dissipative collisions. Crucial in this case is both the precise reconstruction of the emitted $\gamma$-ray direction by the tracking array, as well as the reaction-product-direction measurement by a magnetic spectrometer.

A closer view of the Doppler-broadened lineshapes is given by the projections of the simulated matrices on the $\gamma$ ray-energy axis for $\theta_{\text {rel }}=130^{\circ} \pm 10^{\circ}$ and $170^{\circ} \pm 10^{\circ}$ (panel (f) and (g), respectively), which correspond to the angular coverage of the AGATA array in the GANIL experiment. It is seen that, for lifetime values much longer than the targetcrossing time (i.e., >> $130 \mathrm{fs}$ ), a symmetric lineshape, with the same centroid energy, is observed at all angles (black histograms) after a Doppler-shift correction based on the ion final velocity (i.e., after the target). A considerable lineshape distortion is instead observed for shorter lifetimes, which can be used to obtain a precise estimate of $\tau$ for lifetimes of the order of the target-crossing time (blue lines). The lower limit for lifetime determination is found to be of the order of tens of fs, at which the $\gamma$ line becomes broad and featureless, and significantly shifted in energy (red histograms). As discussed later, crucial for the analysis is also the precise $\gamma$-ray energy determination provided by the $90^{\circ}$ detectors (panel e), which are not affected by the Doppler shift. Altogether, this clearly indicates that the best conditions for precise lifetime determination will be reached by a tracking array with an extended angular coverage, as it is foreseen for AGATA in the coming future [16].

Coming now to the details of the lifetime analysis procedure, here developed, the technique relies on a twodimensional $\chi^{2}$ minimization, in lifetime and transition energy coordinates $\left(\mathrm{E}_{\gamma}, \tau\right)$. The $\chi^{2}$ is calculated considering the measured and simulated Doppler-shift-corrected spectra, over the available $\theta_{\text {rel }}$ angular range. In the presented cases, $\gamma$-ray spectra associated with the three angular ranges $\theta_{\text {rel }}=130^{\circ} \pm 10^{\circ}, 150^{\circ} \pm 10^{\circ}$, and $170^{\circ} \pm 10^{\circ}$ were constructed and simultaneously used in the minimization procedure. The two-dimensional $\chi^{2}$ surface is expected to show a minimum corresponding to the optimal state lifetime and transition energy. The total errors will be obtained by summing contributions from statistics and systematics, the latter arising from uncertainties in the stopping-power parametrization, background subtractions and ion-velocity reconstruction. In the cases discussed below, these total errors 


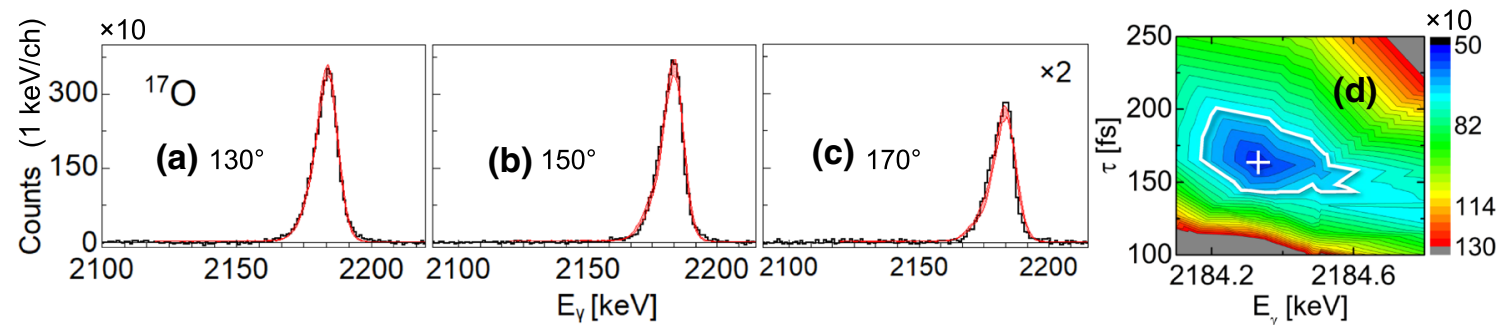

Fig. 18 Doppler-shift corrected AGATA $\gamma$-ray energy spectra (black) and simulated ones (red) in the region of the $2184-\mathrm{keV}$ line of ${ }^{17} \mathrm{O}$, for the angular ranges of $130^{\circ} \pm 10^{\circ}(\mathbf{a}), 150^{\circ} \pm 10^{\circ}(\mathbf{b})$, and $170^{\circ} \pm 10^{\circ}(\mathbf{c})$. Panel d: corresponding two-dimensional $\chi^{2}$ lifetime-energy surface, with the white cross and white contour line indicating the minimum and the uncertainty region, corresponding to $80 \%$ confidence level. The red shaded bands in panel $\mathbf{a}-\mathbf{c}$ are the results of the lineshape simulations performed by varying $\mathrm{E}_{\gamma}$ and $\tau$ within the uncertainty region, i.e., $\left(\mathrm{E}_{\gamma}, \tau\right)=\left(2184.3_{-0.2}^{+0.3}, 159_{-30}^{+40} \mathrm{fs}\right)$
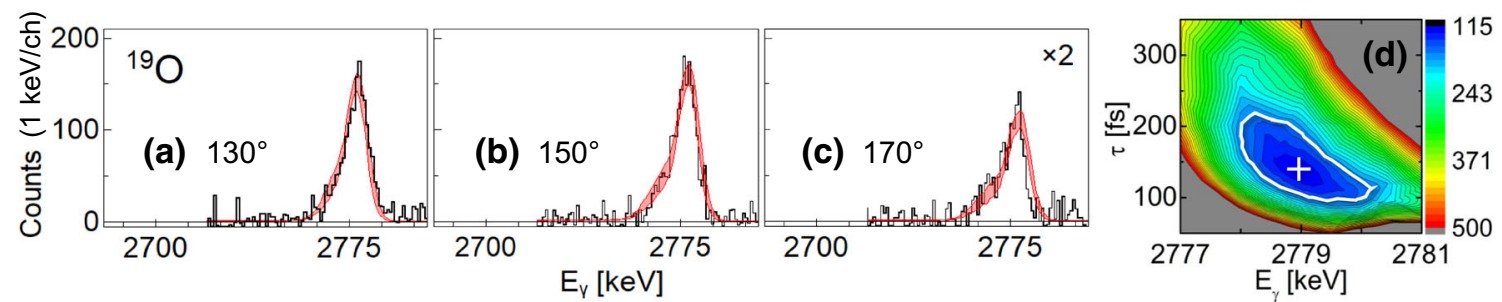

Fig. 19 Same as in Fig. 18 but for the $2779-\mathrm{keV}$ line of ${ }^{19} \mathrm{O}$. The results of the two-dimensional fitting procedure are $\left(\mathrm{E}_{\gamma}, \tau\right)=$ $\left(2779.0_{-0.8}^{+1.0} \mathrm{keV}, 140_{-40}^{+50} \mathrm{fs}\right)$

are equivalent to the uncertainties extracted by considering the region with $80 \%$ confidence level around the $\chi^{2}$ minimum. We note that the lifetime obtained with the present procedure is the cumulative lifetime, i.e., it includes possible contributions from feeding transitions. In all cases here considered, such contribution is negligible, as indicated by the absence of $\gamma$ rays populating the states of interest - these states are fed mostly directly in the transfer reaction or via neutron emission after the transfer process.

Figures 18 and 19 show examples of lifetime analyses for ${ }^{17} \mathrm{O}$ and ${ }^{19} \mathrm{O}$ states for which $\tau$ values of the order of $100 \mathrm{fs}$ are reported in literature [41], i.e., well within the sensitivity range of the present technique. In the first case, the $3055-\mathrm{keV}, 1 / 2_{1}^{-}$state in ${ }^{17} \mathrm{O}$, which is depopulated by a $2184-\mathrm{keV} \gamma$ ray, is considered (see level scheme in Fig. 10). Simulated and experimental $\gamma$-ray spectra are compared in three ranges of the relative angle $\theta_{\text {rel }}: 120^{\circ}-140^{\circ}$, $140^{\circ}-160^{\circ}$ and $160^{\circ}-180^{\circ}$, as shown in panels (a), (b) and (c) of Fig. 18. The corresponding two-dimensional $\chi^{2}$ lifetime-energy surface is reported in panel (d). A welldefined minimum (marked with a white cross) is visible at $\tau=159_{-30}^{+40}$ fs and $E_{\gamma}=2184.3_{-0.2}^{+0.3} \mathrm{keV}$, in agreement, within uncertainty, with the literature values of $\tau=120_{-60}^{+80} \mathrm{fs}$ and $\mathrm{E}_{\gamma}=2184.44(9) \mathrm{keV}$ [41]. The errors are obtained by considering the region around the $\chi^{2}$ minimum, indicated with a white contour in panel (d) $(80 \%$ confidence level, as discussed above). The red shaded bands in panels (a)-(c) are the results of the lineshape simulations performed by varying $\mathrm{E}_{\gamma}$ and $\tau$ within the uncertainty region around the $\chi^{2}$ minimum.

A similar analysis is reported in Fig. 19 for the 2779-keV, $7 / 2^{+}$state in ${ }^{19} \mathrm{O}$, deexcited by a $2779-\mathrm{keV}$ transition (see level scheme in Fig. 11). Also in this case, a well-defined minimum is found in the $\chi^{2}$ map, located at $\tau=140_{-40}^{+50} \mathrm{fs}$ and $\mathrm{E}_{\gamma}=2779.0_{-0.8}^{+1.0} \mathrm{keV}$, in line with previous works (i.e., $\tau=70(26)$ fs [49] and $\tau=117(26)$ fs [48]).

An additional example of lifetime analysis, within the sensitivity range of the present technique, is reported in Ref. [25], for the second $2^{+}$state in ${ }^{20} \mathrm{O}$, located at $4070 \mathrm{keV}$ excitation energy, with a lifetime $\tau=150_{-30}^{+80}$ fs (see later discussion in connection with Fig. 23).

\subsection{Sensitivity limits of the lifetime analysis technique}

As discussed in the previous section, the time range accessible by the present lifetime analysis technique is dictated by the target-crossing time $\mathrm{T}_{\text {cross }}$ of the reaction product (which is about $130 \mathrm{fs}$ for the ${ }^{18} \mathrm{O}+{ }^{181} \mathrm{Ta}$ reaction). The simulation showed that this range spans between $\sim 0.2 \mathrm{~T}_{\text {cross }}$ and $\sim 4 \mathrm{~T}_{\text {cross }}$. Consequently, for lifetime values a few times longer or shorter than $\mathrm{T}_{\text {cross }}$, the hereproposed two-dimensional $\chi^{2}$ minimization procedure, in lifetime-transition energy coordinates $\left(\mathrm{E}_{\gamma}, \tau\right)$, cannot provide a well localized minimum. Rather, a valley extending 

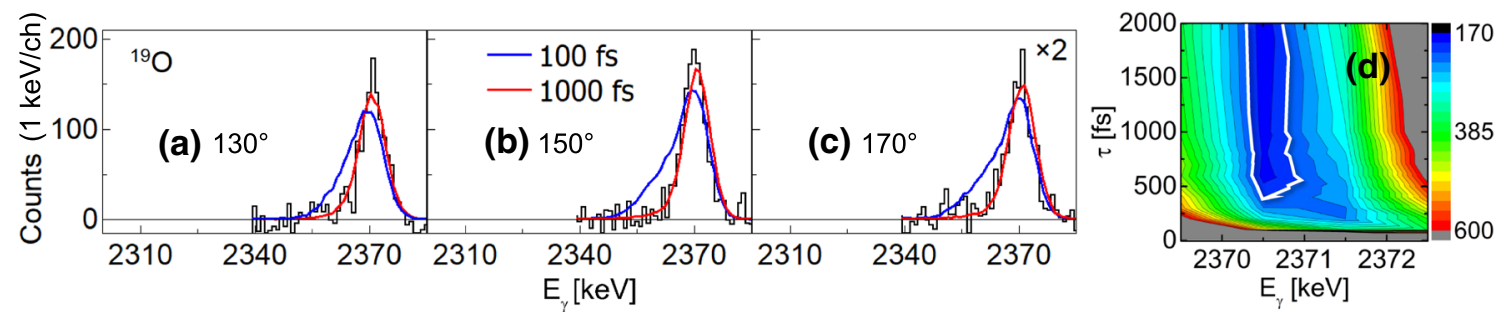

Fig. 20 Same as in Fig. 18 but for the $2371-\mathrm{keV}$ line of ${ }^{19}$ O. Experimental data are shown in black, simulated ones with lifetime values of $100 \mathrm{fs}$ and $1000 \mathrm{fs}$ are displayed by blue and red line, respectively (panels a-c). Panel d: corresponding two-dimensional $\chi^{2}$ surface in $\left(\mathrm{E}_{\gamma}\right.$, $\tau$ ) coordinates, with the white contour line delimiting the uncertainty region (corresponding to $80 \%$ confidence level and giving the lower limit $\tau>400$ fs at $\mathrm{E}_{\gamma}=2370.6_{-0.3}^{+0.5} \mathrm{keV}$ )
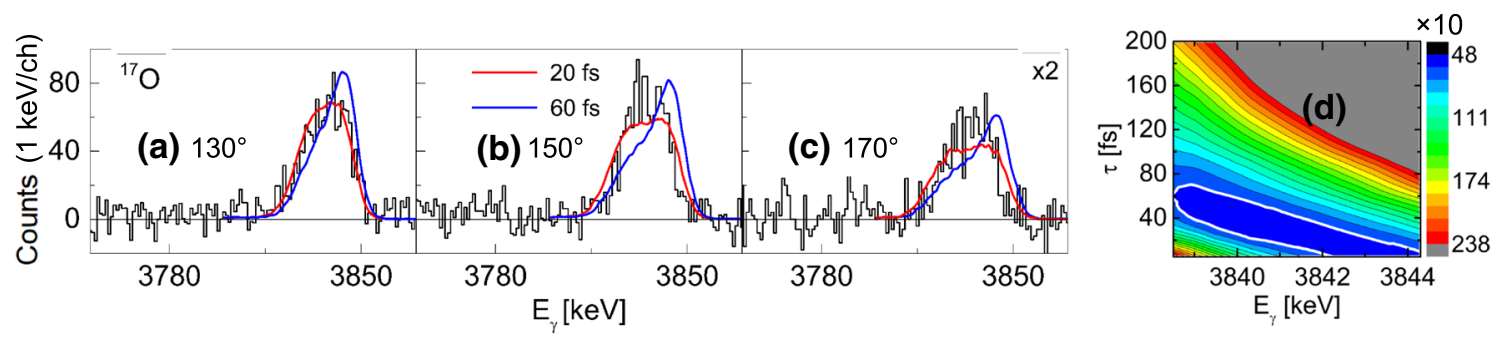

Fig. 21 Same as in Fig. 18 but for the $3842-\mathrm{keV}$ line of ${ }^{17} \mathrm{O}$. Experimental data are shown in black, simulated ones with lifetime values of $20 \mathrm{fs}$ and $60 \mathrm{fs}$ are displayed by red and blue line, respectively (pan-

towards infinitely long lifetimes or reaching the zero value will be obtained.

To illustrate this aspect of sensitivity limit, we consider in Fig. 20 the case of the long-lived $2371-\mathrm{keV}$ state in ${ }^{19} \mathrm{O}$, for which the lower limit $\tau>3.5$ ps is reported in literature [48]. Panels (a), (b) and (c) display the Doppler-shift corrected 2371-keV $\gamma$ ray (deexciting the state), as measured in AGATA in the three angular ranges of $120^{\circ}-140^{\circ}$, $140^{\circ}-160^{\circ}$ and $160^{\circ}-180^{\circ}$, respectively. In all cases, a symmetric Gaussian lineshape is observed, as expected for decays occurring outside the target, at times significantly larger than the target-crossing time. Simulated lineshapes corresponding to $\tau=100$ and $1000 \mathrm{fs}$ are also shown in blue and red, respectively, for comparison. No minimum is obtained in the $\chi^{2}$ map (see Fig. 20d), but a valley is observed, extending from $\tau>400 \mathrm{fs}$, at the $\gamma$-transition energy of $2370.6_{-0.3}^{+0.5} \mathrm{keV}$, which agrees well with the literature value.

Figure 21 shows the case of the short-lived 3842-keV state in ${ }^{17} \mathrm{O}$, for which the upper limit $\tau<26$ fs is reported in literature [50]. The Doppler-shift corrected 3842-keV $\gamma$ ray (deexciting the state) is shown in panels (a), (b) and (c), as measured in AGATA in the three angular ranges of $120^{\circ}-$ $140^{\circ}, 140^{\circ}-160^{\circ}$ and $160^{\circ}-180^{\circ}$, respectively. In all cases, a broad-peak structure is observed around $3835 \mathrm{keV}$, which is consistent with a $\gamma$ emission, inside the target, from a very short-lived state. Simulated lineshapes corresponding to $\tau=$ 20 and 60 fs are also shown in red and blue, respectively, els a-c). Panel d: corresponding two-dimensional $\chi^{2}$ surface in $\left(\mathrm{E}_{\gamma}\right.$, $\tau$ ) coordinates, with the white contour line delimiting the uncertainty region ( $80 \%$ confidence level)

for comparison. Also in this case, no well-defined minimum is found in the $\chi^{2}$ map (see Fig. 21d), but a valley is seen, extending from $70 \mathrm{fs}$ down to $0 \mathrm{fs}$, with a strong dependence on the $\gamma$-ray transition energy. A lifetime $\tau=20_{-20}^{+20}$ fs is obtained if the $\gamma$-transition energy is taken to be 3842.3(4) $\mathrm{keV}$, as reported in literature [41]. This shows the impact of a precise $\gamma$-ray energy determination, which could be best accomplished when the tracking array extends to $90^{\circ}$.

\subsection{Relevance of the AGATA tracking array performances}

The quality of the results of the newly developed lifetime analysis technique depends strongly on the Doppler-shift correction capabilities of the experimental setup. In the case of $\gamma$-ray tracking arrays, such as AGATA, the interaction point is identified with unprecedented precision, with respect to conventional HPGe detectors [14-16]. In a standard configuration of AGATA (at $23.5 \mathrm{~cm}$ from the target center), the angular resolution is around $1^{\circ}$, as a result of the combined use of Pulse Shape Analysis and tracking algorithms. With the use of a magnetic spectrometer, which also offers a resolution of $1^{\circ}$ for the angle detection of the reaction products (as in the case of VAMOS++), the angle between the fragment velocity at the de-excitation point and the $\gamma$-ray direction can be determined with an accuracy of about $1.5^{\circ}$. Such a precision is crucial, together with an accurate measurement of the ion velocity, to perform a Doppler-shift correction which 

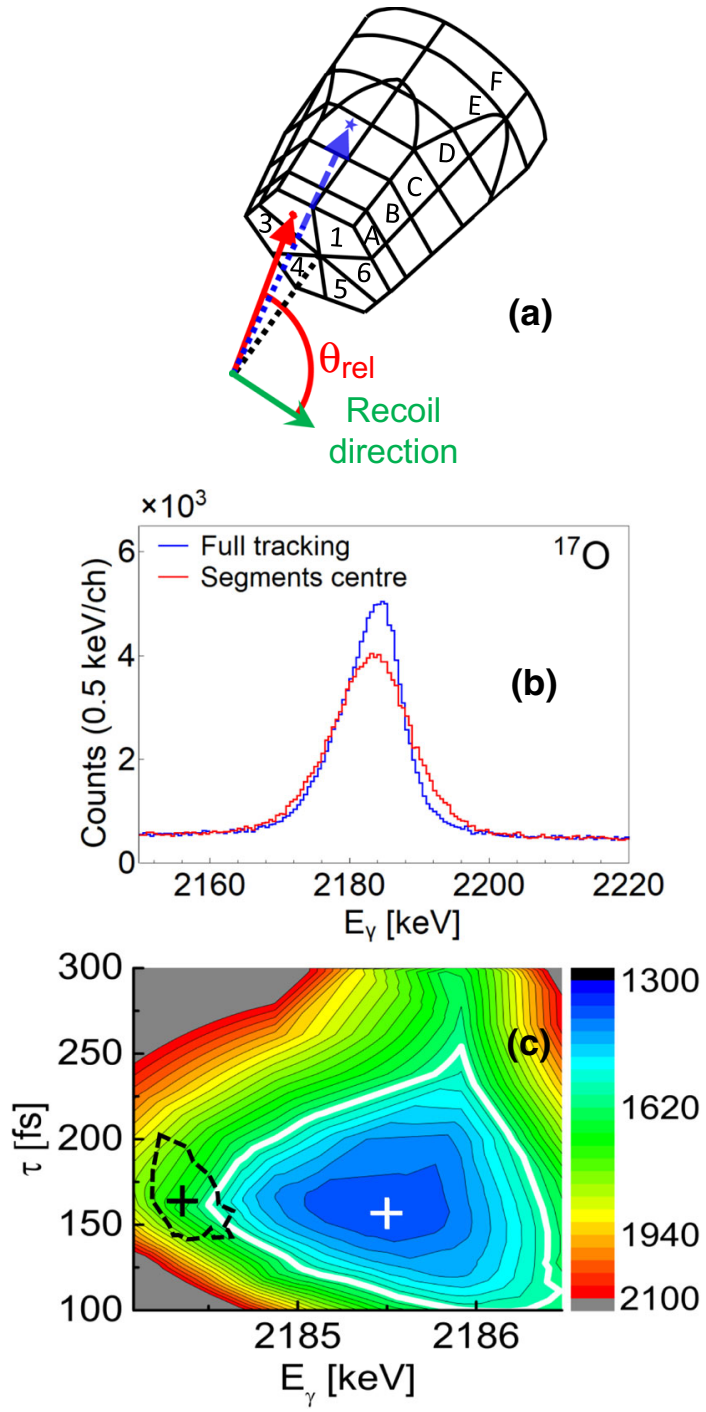

Fig. 22 Panel a: interaction of a $\gamma$-ray, emitted from a recoiling reaction fragment, in a segmented AGATA germanium detector. The real $\gamma$-interaction point is marked by a blue star, the segment center by a red point. Panel b: AGATA Doppler-shift corrected 2184-keV, $1 / 2_{1}^{-} \rightarrow 1 / 2_{1}^{+}, \gamma$ transition of ${ }^{17} \mathrm{O}$, obtained by applying a Dopplershift correction based on the full tracking procedure (blue) or considering the segment center of the AGATA detector (red), as $\gamma$-ray interaction point. Panel c: lifetime-energy $\chi^{2}$ minimization surface for the 2184-keV transition, obtained by considering the segment centers of the AGATA detectors as $\gamma$-ray interaction point. The white cross and contour line indicate the minimum and the uncertainty region. The dashed contour, in black, delimits the uncertainty region obtained with a fulltracking analysis of the AGATA data, as already reported in Fig. 18d

allows for a detailed study of the $\gamma$-ray lineshape, as discussed in this paper.

Figure 22b gives, as an example, the lineshape of the 2184$\mathrm{keV} \gamma$ ray of ${ }^{17} \mathrm{O}$ obtained by determining the $\gamma$-ray interaction points using the full AGATA tracking procedure (blue histogram), or by considering the segment centers (red histogram), as it is done with conventional HPGe detectors (for the determination of the interaction points, see Fig. 22a).

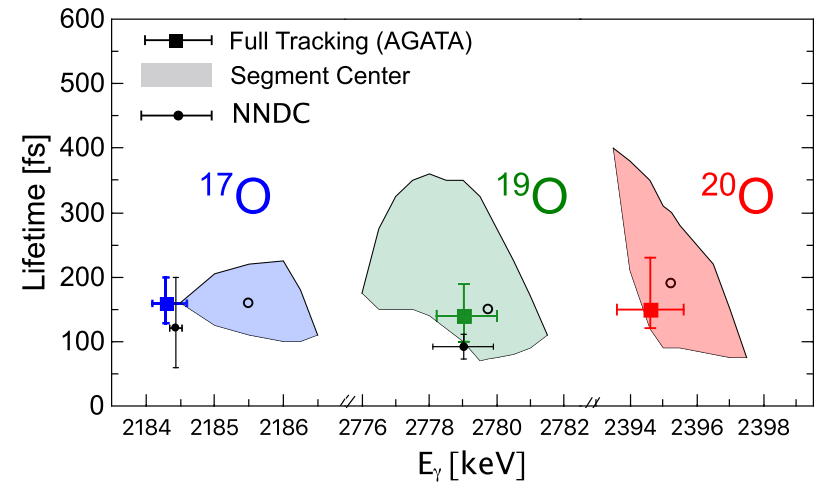

Fig. 23 Lifetime values obtained with the present Monte Carlo technique for the $2184-\mathrm{keV}, 1 / 2^{-}$state of ${ }^{17} \mathrm{O}$ (blue), the $2779-\mathrm{keV}, 7 / 2^{+}$ state of ${ }^{19} \mathrm{O}$ (green), and the $4070-\mathrm{keV}$, second $2^{+}$state of ${ }^{20} \mathrm{O}$ (red), considering the $\gamma$-interaction points extracted with the AGATA fulltracking procedure (square symbols) and the segment centers of the AGATA detectors (contour areas with open circles indicating the best values). For the two test cases of ${ }^{17} \mathrm{O}$ and ${ }^{19} \mathrm{O}$, the NNDC adopted values [41] are given by circles symbols with error bars

In the latter case, the less precise Doppler-shift correction is found to limit significantly the lineshape sensitivity to the lifetime and $\gamma$-ray energy determination. As shown in Fig. 22c, a shallower minimum is obtained in the lifetimetransition energy $\chi^{2}$ surface with respect to Fig. 18d, leading to much larger uncertainties in the final $\mathrm{E}_{\gamma}$ and $\tau$ values. In the specific case of the $2184-\mathrm{keV} \gamma$ ray of ${ }^{17} \mathrm{O}$, the uncertainty region is $\sim 5$ times more extended in $\mathrm{E}_{\gamma}$ and $\sim 2.3$ times wider in $\tau$. Moreover, the $\chi^{2}$ value at the minimum of the two-dimensional map is $\sim 2.5$ times larger than in the AGATA analysis performed with full tracking. A similar behavior of the $\chi^{2}$ map was observed (when the segment centers were considered) in the lifetime analyses of the $7 / 2^{+}$ and second $2^{+}$states in ${ }^{19} \mathrm{O}$ and ${ }^{20} \mathrm{O}$ [25], respectively. Figure 23 displays the results of the lineshape analysis in the case of Doppler-shift corrections based on full tracking (square symbols with error bars) and on interaction positions taken as segment center (contour areas), in the $\left(\mathrm{E}_{\gamma}, \tau\right)$ coordinates. For the two test cases of ${ }^{17} \mathrm{O}$ and ${ }^{19} \mathrm{O}$, the NNDC adopted values [41] are denote by circles with error bars. It is seen that the lifetime and energy determination is quite accurate if the tracking procedure is applied, while the analysis based on the segment-center interaction positions suffers from large uncertainties in both lifetime and $\gamma$-energy coordinates. We note that these uncertainties would be much larger in the case of conventional $\mathrm{HpGe}$ arrays, where individual crystals are typically bigger than AGATA segments.

\section{Conclusions}

A novel Monte Carlo technique has been developed to determine nuclear-state lifetimes of the order of tens-to-hundreds 
femtoseconds (i.e., the target-crossing time), by accurate analysis of Doppler-broadened $\gamma$-ray lineshapes, in lowenergy heavy-ion binary reactions. These reaction processes are characterized by large energy dissipation, leading to complex velocity distributions which do not allow to apply standard lineshape analysis methods. Our procedure makes use of the reaction-product velocity distribution, as measured by a magnetic spectrometer, to reconstruct, via a Monte Carlo simulation, the ion velocity distribution at the reaction instant which is then used to produce simulated Doppler-broadened $\gamma$-ray line shapes, to be compared with the experimental data.

In the present paper, the method is discussed in connection with the analysis of an experiment performed at GANIL with the AGATA+VAMOS+PARIS setup, aiming at the study of excited states lifetimes in neutron-rich $\mathrm{O}, \mathrm{C}$, and $\mathrm{N}$ nuclei [25]. It is demonstrated that the combined use of a magnetic spectrometer and a $\gamma$-tracking array (with few millimeter interaction-point position resolution) becomes essential for the detailed analysis of the $\gamma$-ray lineshapes, resulting in state lifetime determinations. The method will significantly gain in precision when tracking arrays will reach a large angular coverage. The present work clearly shows, as well, that tracking arrays are unique for precision $\gamma$-spectroscopy studies in low-energy reaction regimes, in addition to their powerful application in collisions at relativistic energies, as demonstrated in earlier works $[12,13,16,51-56]$.

The new approach discussed in this work is expected to become an important tool for investigating exotic neutronrich nuclei produced with intense ISOL-type beams in lowenergy heavy-ion binary collisions: it will allow to obtain information on electromagnetic observables which can be used to test the quality of first-principles nuclear structure calculations, complementing common benchmarks based on nuclear-state energies.

Acknowledgements The authors wish to thank the staff of the GANIL Laboratory for providing the beam and for the help given during the experiment. The AGATA collaboration is also acknowledged. This work was supported by the Italian Istituto Nazionale di Fisica Nucleare, by the Polish National Science Centre under Contracts No. 2014/14/M/ST2/00738, No. 2013/08/M/ST2/00257, and No. 2016/22/M/ST2/00269, and by RSF Grant No. 19-42-02014 and by the U.S. Department of Energy, Office of Science, Office of Nuclear Physics, under contract number DE-AC02-06CH11357. This project has received funding from the Turkish Scientific and Research Council (Project No. 115F103) and from the European Union Horizon 2020 Research and Innovation Program under Grant Agreement No. 654002.

Funding Information Open access funding provided by Università degli Studi di Milano within the CRUI-CARE Agreement.

Data Availability Statement This manuscript has no associated data or the data will not be deposited. [Authors' comment: The ownership of data generated by the AGATA spectrometer resides with the AGATA collaboration as detailed in the AGATA Data Policy (http://npg.dl.ac. uk/agata_acc/AGATA_Data\%20Policy.html).]
Open Access This article is licensed under a Creative Commons Attribution 4.0 International License, which permits use, sharing, adaptation, distribution and reproduction in any medium or format, as long as you give appropriate credit to the original author(s) and the source, provide a link to the Creative Commons licence, and indicate if changes were made. The images or other third party material in this article are included in the article's Creative Commons licence, unless indicated otherwise in a credit line to the material. If material is not included in the article's Creative Commons licence and your intended use is not permitted by statutory regulation or exceeds the permitted use, you will need to obtain permission directly from the copyright holder. To view a copy of this licence, visit http://creativecomm ons.org/licenses/by/4.0/.

\section{References}

1. E.M. Burbidge, G.R. Burbidge, W.A. Fowler, F. Hoyle, Rev. Mod. Phys. 29, 547 (1957)

2. P. Moller, B. Pfeiffer, K. Kratz, Phys. Rev. C 67, 055802 (2003)

3. C. Broggini et al., Riv. Nuovo Cim. 42, 103 (2019)

4. W.U. Schröder, J.R. Huizenga, Damped nuclear reactions. In: D.A. Bromley (Ed.), Treatise on heavy-ion science, Vol. 2: fusion and quasi-fusion phenomena (Springer, London, 1985), pp. 113-726

5. R. Kaufmann, R. Wolfgang, Phys. Rev. 121, 192 (1961)

6. J. Wilczynski, Phys. Lett. B 47, 484 (1973)

7. V.I. Zagrebaev, B. Fornal, S. Leoni, W. Greiner, Phys. Rev. C 89, 054608 (2014)

8. A.V. Karpov, V.V. Saiko, Phys. Rev. C 96, 024618 (2017)

9. I. Stefan et al., Phys. Lett. B 779, 456 (2018)

10. U. Köster, Eur. Phys. J. A 15, 255 (2012)

11. P.J. Nolan, J.F. Sharpey-Schafer, Rep. Prog. Phys. 42, 1 (1979)

12. C. Morse et al., Phys. Lett. B 780, 227 (2018)

13. C. Morse et al., Phys. Lett. B 787, 198 (2018)

14. S. Akkoyun et al., Nucl. Instrum. Methods Phys. Res. A 668, 26 (2012)

15. E. Clément et al., Nucl. Instrum. Methods Phys. Res. A 855, 1 (2017)

16. W. Korten et al., Eur. Phys. J. A 56, 137 (2020)

17. S. Paschalis, I.Y. Lee, A.O. Macchiavelli, C.M. Campbell, M. Cromaz, S. Gros et al., Nucl. Instrum. Methods Phys. Res. A 709, 44 (2013)

18. P. Fallon, A. Gade, I.-Y. Lee, Annu. Rev. Nucl. Part. Sci. 66, 321 (2016)

19. M. Rejmund et al., Nucl. Instrum. Methods Phys. Res. A 646, 184 (2011)

20. S.S. Pullanhiotan et al., Nucl. Instrum. Methods Phys. Res. A 593, 343 (2008)

21. A.M. Stefanini et al., Nucl. Phys. A 701, 217c (2002)

22. D. Montanari et al., Eur. Phys. J. A 47, 4 (2011)

23. C.N. Davids, J.D. Larson, Nucl. Instrum. Methods B 40-41, 1224 (1989)

24. D. Bazin et al., Nucl. Instrum. Methods B 204, 629 (2003)

25. M. Ciemała et al., Phys. Rev. C 101, 021303(R) (2020)

26. A. Maj et al., Acta Phys. Pol. B 40, 565 (2009)

27. GANIL Internal report GANIL R 98 02. http://hal.in2p3.fr/ in2p3-00566499

28. M. Vandebrouck et al., Nucl. Instrum. Methods Phys. Res. A 812, 112 (2016)

29. A. Giaz et al., Nucl. Instrum. Meth. Phys. Res. A 729, 910 (2013)

30. G. Gosta et al., Nucl. Instrum. Methods Phys. Res. A 879, 92 (2018)

31. A. Lemasson, Private Communication

32. X. Grave, et al., In: Proceeedings of the $14^{\text {th }}$ Fourteenth IEEENPSS Real Time Conference, 119 (2005) 
33. R. Venturelli, D. Bazzacco, LNL Annual Report No. (2004), p. 220. https://www.lnl.infn.it/ annrep/read_ar/2004

34. L.L.P. Reiter, B. Birkenbach, B. Bruyneel, E. Clement et al., Eur. Phys. J. A 55, 81 (2019)

35. A. Lopez-Martens, K. Hauschild, A. Korichi, J. Roccaz, J.P. Thibaud, Nucl. Instrum. Methods Phys. Res. A 533, 454 (2004)

36. B. Bruyneel et al., Nucl. Instrum. Methods Phys. Res. A 608, 99 (2009)

37. B. Bruyneel et al., Eur. Phys. J. A 49, 61 (2013)

38. AGATA software package. https://atrium.in2p3.fr/

39. O.B. Tarasov, D. Bazin, Nucl. Instrum. Methods Phys. Res. B 266, 4657 (2008)

40. R.B. Firestone, Z. Revay, Phys. Rev. C 93, 044311 (2016)

41. National Nuclear Data Center [Online]. https://www.nndc.bnl.gov/ nudat $2 /$

42. J. Ziegler, J. Biersack, U.U. Littmark, The Stopping Power and Range of Ions in Solids, vol. 1 (Pergamon, New York, 1984)

43. R. Anne et al., Nucl. Instrum. Methods Phys. Res. B 34, 295 (1988)
44. H. Geissel et al., Nucl. Instrum. Methods B 195, 3 (2002)

45. M. Labiche, et al., AGATA GEANT4 Simulations for AGATA@GANIL. http://npg.dl.ac.uk/svn/agata/

46. C. Stahl, J. Leske, M. Lettmann, N. Pietralla, Comp. Phys. Commun. 214, 174 (2017)

47. C. Michelagnoli et al., AIP Conf. Proc. 1484, 281 (2012)

48. F. Hibou, P. Fintz, B. Rastegar, A. Gallmann, Nucl. Phys. A 171, $603(1971)$

49. C. Broude et al., Nucl. Phys. A 161, 241 (1971)

50. T.K. Alexander, C. Broude, A.E. Litherland, Nucl. Phys. 53, 593 (1964)

51. R. Avigo et al., Phys. Lett. B 811, 135951 (2020)

52. A. Bracco, F.C.L. Crespi, E.G. Lanza, Eur. Phys. J. A 51, 99 (2015)

53. Z. Podolyak et al., Phys. Rev. Lett. 117, 222302 (2016)

54. A. Boso et al., Phys. Lett. B 797, 1485 (2019)

55. A. Gade et al., Phys. Rev. Lett. 112, 112503 (2014)

56. S.R. Stroberg et al., Phys. Rev. C 90, 034301 (2014) 